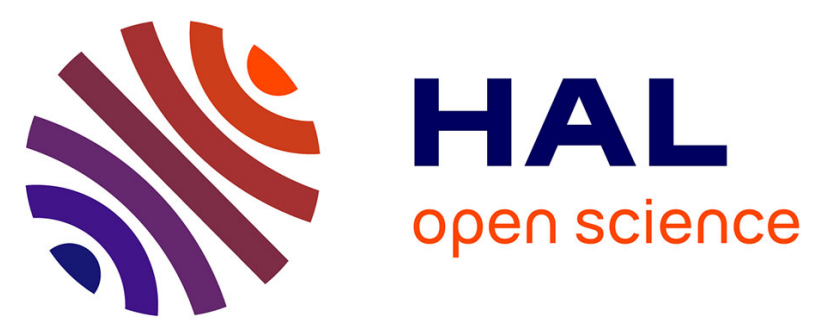

\title{
Construction et négociation de l'identité nationale. Une étude comparatiste des programmes et des manuels scolaires d'histoire pour le primaire en France et aux États-Unis (1980-2010)
}

Rachel Hutchins

\section{To cite this version:}

Rachel Hutchins. Construction et négociation de l'identité nationale. Une étude comparatiste des programmes et des manuels scolaires d'histoire pour le primaire en France et aux États-Unis (19802010). Histoire@Politique: revue du Centre d'histoire de Sciences Po, 2015, 26. hal-01525675

\section{HAL Id: hal-01525675 \\ https://hal.univ-lorraine.fr/hal-01525675}

Submitted on 22 May 2017

HAL is a multi-disciplinary open access archive for the deposit and dissemination of scientific research documents, whether they are published or not. The documents may come from teaching and research institutions in France or abroad, or from public or private research centers.
L'archive ouverte pluridisciplinaire HAL, est destinée au dépôt et à la diffusion de documents scientifiques de niveau recherche, publiés ou non, émanant des établissements d'enseignement et de recherche français ou étrangers, des laboratoires publics ou privés. 
Rachel Hutchins, "Construction et négociation de l'identité nationale. Une étude comparatiste des programmes et des manuels scolaires d'histoire pour le primaire en France et aux États-Unis », Histoire@Politique, n² 26, maiaoût 2015, www.histoire-politique.fr

\title{
Construction et négociation de l'identité nationale. Une étude comparatiste des programmes et des manuels scolaires d'histoire pour le primaire en France et aux États-Unis (1980-2010)
}

\author{
Rachel Hutchins
}

\section{Enjeux et définitions}

L'enseignement public est investi de missions semblables en France et aux États-Unis depuis ses débuts. Chargée de former des citoyens et d'assurer l'unité nationale, l'école constitue dans les deux pays un lieu privilégié pour forger une définition de l'identité nationale. L'histoire, la géographie et l'éducation civique sont des matières particulièrement sujettes aux changements idéologiques suivant les évolutions de l'opinion publique.

La présente étude compare les manuels et, dans une moindre mesure, les programmes de ces matières en France et aux États-Unis de 1980 à 2010. La comparaison de ces deux pays à cette époque particulière est instructive à plusieurs égards. Les deux pays reposent sur des mythes fondateurs semblables : des Étatsnations nés du siècle des Lumières, conçus officiellement comme "civiques », où l'appartenance au corps national ne dépend pas de sa généalogie mais de son choix. Les deux pays se sont dotés, dans le passé, d'une «mission civilisatrice » qui consistait à promouvoir des valeurs tenues pour universelles, fondée sur une croyance en la supériorité et la particularité nationales.

La France et les États-Unis sont également les lieux de débats similaires notamment depuis les années 1960 concernant l'historiographie, l'épistémologie, la place des minorités ethniques et des femmes dans la nation et le récit national. Ces débats ont accompagné des changements démographiques et sociaux, avec l'arrivée dans les deux pays de nombreux immigrés provenant de régions extra-européennes et, aux États-Unis, le mouvement pour les droits civiques des Afro-américains ${ }^{1}$. Les deux

\footnotetext{
${ }^{1}$ Dans un même temps, l'élargissement de l'Union européenne contribue également au malaise français quant à la préservation du caractère et du pouvoir nationaux. Bien que la question européenne fasse partie du contexte de la présente étude, nous nous concentrons ici davantage sur les questions identitaires liées à l'immigration. D’autres études montrent comment les politiques culturelles et éducatives françaises ont affirmé la spécificité française dans un cadre avant tout national en réponse à l'européanisation de ces domaines. Voir Vincent Martigny, Le Nationalisme culturel français. Récit national et usages politiques de la culture en France (1981-1995), thèse de doctorat, Institut d'études politiques de Paris, 2012 ; Géraldine Bozec, Les héritiers de la République. Éduquer à la citoyenneté à l'école dans la France d'aujourd'hui, thèse de doctorat, Institut d'études politiques de Paris, 2010, p. 229-263 ; Laurent Wirth, « Le pouvoir politique et l'enseignement de l'histoire: L'exemple des finalités civiques assignées à cet enseignement en France depuis J ules Ferry », Histoire@Politique, $\mathrm{n}^{\circ} 2$,
} 
Rachel Hutchins, "Construction et négociation de l'identité nationale. Une étude comparatiste des programmes et des manuels scolaires d'histoire pour le primaire en France et aux États-Unis », Histoire@Politique, n² 26, maiaoût 2015, www.histoire-politique.fr

pays ont connu des changements pédagogiques sur la forme comme sur le fond depuis cette époque, ainsi qu'une réaction contre cette nouvelle pédagogie. Dans les deux pays, ces processus ont engendré une réflexion sur la nature et les objectifs de l'enseignement de l'histoire qui s'est amplifiée à partir du début des années 1980. Dans les deux cas, cette réflexion est souvent définie en termes de «crise» de l'enseignement, et elle concerne à la fois la grandeur nationale, l'avenir économique et moral du pays ainsi que la définition de l'identité nationale. Dans ce contexte d'évolutions des origines nationales et ethniques de la population, ainsi que des changements épistémologiques et idéologiques de la pratique enseignante, il est révélateur d'examiner comment se construit l'identité nationale dans la version officielle véhiculée par l'enseignement du primaire au cours des trente dernières années ${ }^{2}$.

Cette étude nécessite une prise en compte de quelques notions dont la définition est souvent contestée. Il est donc utile d'en préciser préalablement l'utilisation qui en sera faite ici. Le terme de «multiculturalisme » peut être employé par ses détracteurs comme synonyme de « communautarisme ». Ici, il reprend l'usage le plus consensuel qui en est fait aux États-Unis, dont les partisans prônent une vision d'un pays uni autour d'une culture commune qui a pour base des valeurs civiques. Cette culture nationale partagée exige néanmoins de revoir le récit historique traditionnel et la définition de la culture nationale, afin d'y intégrer les expériences et les points de vue des minorités ethniques et des femmes. Ce multiculturalisme repose sur l'idée que les individus sont dotés d'appartenances multiples: on peut être Américain tout en revendiquant ses origines ethniques (en plus d'autres types d'identité) ${ }^{3}$.

Cette vision n'est pas incompatible avec le «nationalisme » dans l'acception retenue ici : le nationalisme consiste à affirmer que l'État-nation est la seule forme de gouvernement légitime. Pour un nationaliste, un peuple se reconnaissant comme formant une nation (définie selon des critères variables, qui peuvent englober des dimensions culturelles, historiques, géographiques, ethniques, économiques, religieuses, etc.) doit être gouverné par un Etat dont les frontières correspondent à celles de la nation. Le nationalisme fait ainsi partie des idéologies les plus répandues de l'époque moderne ${ }^{4}$. Si cette conception du nationalisme englobe l'utilisation qui en est faite plus couramment en français, elle est plus vaste. Le nationalisme peut être de nature plus ou moins inclusive ou exclusive ; il peut être plus ou moins fondé sur des facteurs d'appartenance liés à un groupe ethnique, à des critères culturels ou encore d'ordre civique; il peut reposer plutôt sur un projet d'avenir construit ensemble par tous ceux qui souhaitent s'y associer ou sur un passé mythique qui détermine qui sont les « vrais » membres de la communauté nationale. Dans les pays européens et

2007, http://histoire-politique.fr/index.php?numero=02\&rub=dossier\&item=14 (lien consulté le 1 er mars 2015).

2 Bien entendu, il existe aussi des différences historiques considérables entre la France et les États-Unis. Les différences qui engendrent des contrastes saillants pour la présente étude seront évoquées au cours de cet article. Pour le moment, l'essentiel est de retenir les ressemblances qui rendent la comparaison féconde.

3 James A. Banks, Multicultural Education, Transformative Knowledge, and Action: Historical and Contemporary Perspectives, New York, Teachers College Press, « Multicultural Education Series », 1996 ; Gary B. Nash et al., History on Trial: Culture Wars and the Teaching of the Past, New York, Vintage Books, 2000 [1997].

4 John Breuilly, Nationalism and the State, Chicago, University of Chicago Press, 2e édition, 1993 [1982] ; Ernest Gellner, Nationalism, London, Weidenfeld \& Nicholson, 1997. 
Rachel Hutchins, "Construction et négociation de l'identité nationale. Une étude comparatiste des programmes et des manuels scolaires d'histoire pour le primaire en France et aux États-Unis », Histoire@Politique, n² 26, maiaoût 2015, www.histoire-politique.fr

américains, on retrouve généralement (dans des proportions différentes) des partisans de ces conceptions différentes de la nation, qui contestent continuellement le sens de leur identité nationale. Toutefois, à partir du moment où ils affirment que la nation est le groupe d'appartenance primaire, et que celui-ci correspond (ou doit correspondre) à la gouvernance de l'État, tous ces partisans adhèrent à un système nationaliste et emploient certains discours et procédés en commun, par lesquels les États-nations se perpétuent. Il s'agit évidemment d'une théorie dont la mise en pratique n'est pas aussi claire, les frontières - qu'elles soient territoriales, humaines ou culturelles - des nations étant floues, changeantes et historiquement construites. L'identité nationale n'est pas fixée une fois pour toutes, mais elle est sans cesse renouvelée au travers d'un processus de contestation et de controverses qui aboutissent à des renégociations et des redéfinitions 5 .

Ainsi, pour les partisans américains d'un multiculturalisme nationaliste, la diversité et le respect des différences culturelles et des points de vue divergents s'inscrivent dans l'identité commune. En revanche, pour certains conservateurs, l'accent doit être mis davantage sur l'unité et les aspects « positifs » de l'identité nationale, ce qui, pour eux, exclut le fait d'accentuer les injustices du passé. Ils prônent une vision d'un pays uni et égalitaire où l'évocation des inégalités liées aux divisions ethniques, de sexe ou de classe perpétue des divisions. Ce point de vue s'est développé notamment depuis les années 1960 en réaction au multiculturalisme croissant, et il s'est réaffirmé après les attaques du 11 septembre $2001^{6}$.

En France, les questions ne se posent pas tout à fait dans les mêmes termes. Le présent article est axé sur l'examen des instructions officielles concernant l'enseignement et à celui des manuels scolaires. Toutefois, il convient de souligner que les débats sur le rôle de l'enseignement de l'histoire et des discussions généralisées plus ou moins simultanées concernant l'identité nationale ont un impact important sur les programmes. Le programme d'histoire de CM2 de 1980 a été le premier depuis 1945 ; dans les années 1970, il n'y avait pas de programme d'histoire, mais cette matière faisait partie des disciplines dites « d'éveil ». Suivant les travaux en psychologie développementale de J ean Piaget ainsi que l'historiographie de l'école des Annales, il s'agissait alors non plus d'enseigner un récit sur l'histoire nationale ni de se servir de l'enseignement de l'histoire pour transmettre la mémoire nationale, mais de laisser les élèves du primaire découvrir le passé (local comme celui des civilisations lointaines) par les sources primaires. Une série de rapports, de colloques et de débats rédigés à la fin des années 1970 remet questionne cette pédagogie « d'éveil » et l'état de l'enseignement de l'histoire en France. D’avis général (historiens, politiques, enseignants du primaire et du secondaire...), les écoles n'enseignaient plus l'histoire française. Un consensus émergea qui mettait l'accent sur les objectifs civiques de l'enseignement de l'histoire, prônant l'idée que l'histoire

\footnotetext{
${ }^{5}$ John Hutchinson, Nations as Zones of Conflict, London, Thousand Oaks, New Delhi, Sage Publications, 2005; Anthony D. Smith, Myths and Memories of the Nation, Oxford, Oxford University Press, 1999.

${ }^{6}$ Il est à noter que cette période correspond au regain du conservatisme dans la politique étatsunienne, et que la position réactionnaire concernant l'identité nationale constituait - et continue à constituer une partie intégrante du message de la droite conservatrice. Voir par exemple: David Farber, The Rise and Fall of Modern American Conservatism: a Short History, Princeton, NJ, Princeton University Press, 2010 ; Eric P. Kaufmann, The Rise and Fall of Anglo-America, Cambridge, MA, Harvard University Press, 2004.
} 
Rachel Hutchins, "Construction et négociation de l'identité nationale. Une étude comparatiste des programmes et des manuels scolaires d'histoire pour le primaire en France et aux États-Unis », Histoire@Politique, n ${ }^{\circ} 26$, maiaoût 2015, www.histoire-politique.fr

scolaire devait maintenir la mémoire nationale collective et se focaliser sur les valeurs de la nation à travers l'histoire politique?

Ainsi, le programme de CM2 de 1980 marque un retour à de l'histoire « événementielle » associée à une visée nationaliste. Dans cet esprit, il mentionne l'objectif de développer par l'enseignement de l'histoire et de la géographie le « sentiment d'appartenir à une collectivité nationale ${ }^{8}$ ». Les programmes successifs évoluent dans le sens de ce retour au lien entre histoire scolaire et identité nationale, mettant davantage l'accent sur la culture nationale.

Toutefois, cette attention accrue à l'enseignement de l'histoire et à ses liens avec la culture nationale entraîne un questionnement sur la nature de cette identité collective. Celui-ci se fait parallèlement à une attention médiatique et politique plus générale sur la place des immigrés récents dans la société française et des ramifications pour la cohésion nationale ${ }^{9}$. Au début des années 1980, quelques éminents historiens, tels que Mona Ozouf ou Philippe J outard, ont prôné un certain pluralisme culturel ${ }^{10}$. Toutefois, comme l'a montré Patricia Legris, sous le ministère de Jean-Pierre Chevènement (1984-1986), l'accent a été mis sur un assimilationnisme républicain traditionnel, qui considérait le pluralisme culturel comme dangereux pour l'unité nationale ${ }^{11}$. Cette position assimilationniste a reçu l'approbation d'une grande majorité des enseignants, des parents et des élèves ${ }^{12}$. Comme le démontre la politologue Elaine Thomas, cette insistance sur l'assimilation à une culture commune française visait à apaiser les tensions entre les différentes communautés ethniques (y compris religieuses). Selon Elaine Thomas, le gouvernement cherchait ainsi à montrer son engagement pour l'intégration des communautés issues de l'immigration et la préservation de la « culture française »; il s'agissait de rassurer ceux qui pouvaient se sentir attirés par la politique du Front national et de freiner, par ce biais, la montée de l'intolérance ${ }^{13}$. Dans les années 1990 se sont développées des multiples associations commémoratives réclamant que soit reconnue l'histoire de la colonisation et de l'esclavage. C'est à cette époque que l’histoire de la colonisation a été institutionnalisée en étant enseignée à l'université ${ }^{14}$.

\footnotetext{
${ }^{7}$ Patrick Garcia et J ean Leduc, L'enseignement de l'histoire en France de l'Ancien Régime à nos jours, Paris, Armand Colin, 2003, p. 197; 205-206; 238; Patricia Legris, Qui écrit les programmes d'histoire?, Grenoble, Presses universitaires de Grenoble, 2014, p. 95-110 ; Patricia Legris, «Les Programmes d'histoire en France: la construction progressive d'une "citoyenneté plurielle" (19802010) », Histoire de l'éducation, $n^{\circ} 126,2010$, p. 124.

8 Cycle moyen, Objectifs, programmes et instructions, Histoire-géographie, «Objectifs », arrêté du 16 juillet 1980, RLR 514-4, p. 44.

9 Gérard Noiriel, Population, immigration et identité nationale en France (XIX e-XX e siècles), Paris, Hachette, 1992 ; Gérard Noiriel, À quoi sert l'identité « nationale», Marseille, Agone, 2007; Elaine R. Thomas, Immigration, Islam, and the Politics of Belonging in France: A Comparative Framework, Philadelphia, University of Pennsylvania Press, 2012.

${ }_{10}$ Patricia Legris « Les Programmes... », op. cit., p. 129.

11 Ibid., p. 129-130.

12 Abby Waldman, « The Politics of History Teaching in England and France during the 1980s », History Workshop J ournal, n 68, 2009, p. 199-221. Géraldine Bozec, Les héritiers de la République. Éduquer à la citoyenneté à l'école dans la France d’aujourd'hui, thèse de doctorat, Institut d'études politiques de Paris, 2010, p. 323.

${ }^{13}$ Elaine Thomas, Immigration..., op. cit., p. 150.

14 Geoffrey de Laforcade, «"Foreigners", Nationalism and the "Colonial Fracture" : Stigmatized Subjects of Historical Memory in France », International J ournal of Comparative Sociology, tome 47, $\mathrm{n}^{\circ} 3-4$, 2006, p. 227.
} 
Rachel Hutchins, "Construction et négociation de l'identité nationale. Une étude comparatiste des programmes et des manuels scolaires d'histoire pour le primaire en France et aux États-Unis », Histoire@Politique, n ${ }^{\circ} 26$, maiaoût 2015, www.histoire-politique.fr

On peut distinguer deux axes de la culture française telle qu'elle a été représentée dans les programmes scolaires au cours de la période étudiée: d'un côté, une dimension civique exige la connaissance de la République avec ses valeurs et ses institutions ; de l'autre, une dimension culturelle met l'accent sur la langue française, l'art, l'histoire et la littérature :

« La connaissance de notre héritage historique, l'assimilation du patrimoine politique et culturel de la France, la découverte des richesses de notre peuple et de notre pays sont indispensables à la formation du citoyen français. L'histoire et la géographie participent à l'apparition chez l'élève de la conscience nationale ${ }^{15}$. 》

Les programmes, en devenant plus longs et détaillés au fur et à mesure des années (en 2008, les responsables ont toutefois choisi d'en réduire le contenu par souci de lisibilité16), tendent à renforcer ces objectifs civiques de l'enseignement par de plus amples précisions concernant les contenus à transmettre. Ce changement ne concerne pas uniquement les programmes d'histoire mais l'intégralité des programmes du primaire. La présentation de l'apprentissage de la langue française est éclairante sur ce point. En 1980, on insistait sur l'importance de l'apprentissage de la langue française dans une optique pédagogique comme base pour la scolarité future. À partir de 1985, les programmes soulignent, davantage que les précédents, l'aspect culturel et patrimonial de la langue nationale. Celui de 1985 explique par exemple :

«Aux enfants pour qui le français n'est pas la langue d'origine, l'école fournit un moyen d'intégration à la communauté française, ainsi qu'une culture toujours ouverte à la diversité. Pour tous les enfants, quelle que soit leur origine, la langue française dans sa richesse et sa rigueur s'apprend à l'école. La pratique réglée de la langue [...] engendre un plaisir esthétique indispensable à l'apprentissage initial.

« Tout doit donc être mis en ouvre pour développer chez l'élève à la fois le goût de la lecture et l'ensemble des capacités qu'elle exige ; à cette condition, l'enseignement de la langue française lui permet de disposer d'une langue de communication riche et diverse, mais également de s'approprier la culture vivante de l'humanité17. 》

Une conception culturelle de l'identité nationale domine ainsi les programmes récents. Au lieu de définir les objectifs dans des termes pédagogiques, comme le faisaient les instructions officielles relatives aux activités "d'éveil », ou de viser à transmettre surtout des connaissances d'ordre civique (sur les valeurs et le fonctionnement de l'État par exemple), ces programmes tendent à transmettre un patrimoine culturel (y compris historique) afin de fédérer la communauté nationale. En cela, ils rejoignent les programmes des États-Unis. Nous examinerons les résultats de ces évolutions plus en détail ci-après.

Il existe bien entendu des différences importantes entre les systèmes éducatifs en question. Autant le système français est centralisé, autant il est décentralisé aux Etats-Unis, chaque État définissant ses propres programmes, règlements de fonctionnement, budgets, etc. Toutefois, grâce aux manuels scolaires, on peut plus facilement comparer les situations dans les deux pays. Pour le primaire, il n'existe

\footnotetext{
${ }^{15}$ Horaires, programmes et instructions pour l'école élémentaire, « Nature et objectifs », arrêté du 23 avril 1985, Histoire et géographie, RLR 514-4, p. 17.

16 « Horaires et programmes d'enseignement de l'école primaire, 2008 », Bulletin officiel de l'Éducation nationale, numéro spécial 3, 2008, p. 10.

${ }^{17}$ Horaires..., arrêté du 23 avril 1985, Français, RLR 514-4, op. cit., p. 5.
} 
Rachel Hutchins, "Construction et négociation de l'identité nationale. Une étude comparatiste des programmes et des manuels scolaires d'histoire pour le primaire en France et aux États-Unis », Histoire@Politique, n² 26, maiaoût 2015, www.histoire-politique.fr

aux États-Unis que quatre grandes maisons d'édition qui vendent une ou deux séries de manuels à l'échelle nationale. Ces ouvrages créent ainsi une sorte d'unité nationale. L'importance des manuels dans l'enseignement américain est renforcée par le fait que les programmes américains sont, de manière générale, moins extensifs qu'en France, et se présentent sous forme de listes de compétences et d'exemples de connaissances préconisées. En outre, le système américain ne comprend pas d'inspecteurs ni (pour l'instant) d'examens à grande échelle pour l'école primaire en histoire. Ainsi, les manuels sont d'une grande importance pour déterminer le contenu dans les salles de classe ${ }^{18}$.

Pour les programmes, les manuels à analyser et les débats en amont de la publication de ces ouvrages, nous centrerons l'analyse sur le cas du Texas. Cet État exerce une influence inégalée sur le contenu des manuels scolaires américains; ses écoles publiques comptent plus de cinq millions d'élèves et établit une liste d'ouvrages dont il assure l'achat pour toutes les écoles. Par ailleurs, le processus de sélection des manuels y est fortement politisé car l'État donne un pouvoir considérable à ses citoyens qui peuvent influencer le contenu des programmes et des manuels. J usqu'à présent, les manuels vendus au Texas sont aussi largement vendus à l'échelle nationale ${ }^{19}$.

En France comme aux États-Unis, l'histoire nationale est abordée vers la fin de l'école primaire. Ces années sont formatrices pour l'identité nationale des élèves. C'est à cette période que se posent les bases de la socialisation, que l'enfant développe le sentiment d'appartenance à une nation, et qu'il se fait une idée de la nature de cette communauté. Même si l'éducation plus nuancée du secondaire peut corriger certaines représentations trop simplistes acquises à l'école primaire, les bases de l'identité nationale sont acquises à cet âge ${ }^{20}$. Dans les deux pays, les manuels sont non seulement un outil pédagogique, mais également un produit commercial, car ils sont publiés par des maisons d'édition indépendantes des gouvernements. Bien entendu, la politique officielle en définit en partie les contenus. Les manuels français doivent se conformer aux programmes établis sous l'autorité du ministère de l'Éducation nationale. De même, les manuels étatsuniens doivent être conformes aux programmes des différents États pour y être sélectionnés, bien qu'ils ne soient pas forcément tenus de suivre les programmes dans leur intégralité. Le Texas, par exemple, exige qu'un manuel inclue au moins la moitié du contenu précisé dans le programme de l'État. Ceci permet aux maisons d'édition de concilier les critères de plusieurs programmes afin de vendre dans un maximum d’États. Au-delà de ces détails, en France comme aux États-Unis, même si les programmes d'histoire listent

\footnotetext{
18 James W. Loewen, Lies My Teacher Told Me: Everything Your American History Textbook Got Wrong, New York, Touchstone, 1995, p. 367; Chester Finn et Diane Ravitch, The Mad, Mad World of Textbook Adoption, Thomas B. Fordham Institute, 29 sept. 2004, http://edex.s3-us-west2.amazonaws.com/publication/pdfs/Mad\%20World Test2 8.pdf (lien consulté le 25 février 2015). Malie Montagutelli, Histoire del'enseignement aux États-Unis, Paris, Belin, 2000, p. 7.

${ }_{19}$ Philip G. Altbach, Gail P. Kelly, Hugh G. Petrie, Lois Weis (eds.), Textbooks in American Society: Politics, Policy, and Pedgagogy, Albany, State University of New York Press, 1991, p. 117-119 ; Joan Delfattore, What J ohnny Shouldn't Read: Textbook Censorship in America, New Haven, CT. ; London, Yale University Press, 1992, p. 121, 138, 142 ; C. Finn et D. Ravitch, « The Mad, Mad World...», op. cit. ${ }^{20}$ Orit Ichilov (ed.), Political Socialization, Citizenship Education, And Democracy, New York, Teachers College Press, 1990 ; Bruce Van Sledright, « Narratives of Nation-State, Historical Knowledge, and School History Education », Review of Research in Education, tome 32, 2008, p. 11. Voir également Géraldine Bozec, Les héritiers..., op. cit., p. 10-11.
} 
Rachel Hutchins, "Construction et négociation de l'identité nationale. Une étude comparatiste des programmes et des manuels scolaires d'histoire pour le primaire en France et aux États-Unis », Histoire@Politique, n² 26, maiaoût 2015, www.histoire-politique.fr

des événements et des périodes à enseigner, ils laissent une certaine marge de manœuvre aux éditeurs. Ces derniers sont libres de choisir des approches différentes pour les sujets à traiter, car tout n'est pas explicité.

Aux États-Unis, la rédaction des manuels est un processus opaque. De manière générale, ceux-ci portent tous des noms d'universitaires à titre d'auteurs et de consultants. Une grande partie de ces personnes sont des experts en sciences de l'éducation, plutôt que des historiens ou des géographes. Néanmoins, de nombreux chercheurs affirment que les « auteurs » dont les noms figurent sur les manuels ne participent en réalité que très peu à la rédaction des ouvrages ${ }^{21}$. Ces derniers sont ce qu'on appelle souvent des « managed textbooks » : le directeur de publication n'est généralement pas celui dont le nom figure dans l'ouvrage, mais une personne anonyme officiant au sein de la maison d'édition. Celui-ci coordonne le projet et exerce l'autorité ultime sur le contenu des ouvrages. Il est donc difficile de savoir qui les rédige véritablement. Par ailleurs, les manuels doivent intégrer les modifications exigées par les élus du Conseil scolaire de l'État du Texas où, en règle générale, les enseignants sont minoritaires ${ }^{22}$. En revanche, en France, bien que les éditeurs des manuels scolaires communiquent avec des associations diverses et que les programmes soient également sujets à des controverses et des débats publics, les manuels et les programmes d'histoire-géographie sont écrits de manière plus transparente qu'outre-Atlantique. Les équipes réunies par les éditeurs sont composées de chercheurs universitaires en histoire et en géographie, des professeurs des écoles, des inspecteurs et des formateurs en IUFM/ ESPE ${ }^{23}$. Ces différences dans les processus de production des manuels scolaires ont des effets sur leur contenu. Aux États-Unis, l'histoire scolaire représente davantage un compromis entre l'histoire universitaire et l'histoire telle que le grand public la conçoit, alors que l'histoire scolaire en France s'aligne davantage sur les exigences de la discipline savante.

Cette étude analyse trente-six manuels scolaires français et américains d'histoire, géographie et éducation civique pour le CM1 et CM2 en France et l'équivalent du CM2 aux États-Unis, et les programmes à partir desquels ils ont été conçus. Ainsi seront analysés des manuels français dont la publication a suivi l'entrée en vigueur des programmes de 1985, 1995, 2002 et 2008, et des ouvrages étatsuniens qui correspondent aux processus de sélection des manuels scolaires au Texas en 1982, 1997 et 2003 - dernière année où le Texas a sélectionné de nouveaux livres. Les manuels des années 1980 sont donc les premiers publiés après les premières réformes multiculturalistes aux États-Unis; ils succèdent aux débats épistémologiques des années 1960 et 1970 dans les deux pays. Depuis cette époque, les deux pays ont continué d'être en proie à des polémiques continues concernant l'intégration des immigrés et des populations issues de l'immigration; aux ÉtatsUnis, l'inégalité persistante entre les Américains d'origine européenne et ceux

\footnotetext{
${ }^{21}$ Pour citer quelques sources parmi la pléthore qui existe : Sherry Keith, "The Determinants of Textbook Content", dans P. Altbach et alii (eds.), Textbooks in American Society, op. cit., p. 48 ; James Loewen, Lies..., op. cit., p. 282-283 ; FrancesFitzGerald, America Revised, New York, Vintage, 1979, p. 20-26; Michael W. Apple, "The Culture and Commerce of the Textbook," in Michael Apple et Linda ChristianSmith (eds), The Politics of the Textbook, New York, Routledge, 1991, p. 30-31.

${ }^{22}$ Le conseil actuel, par exemple, compte sept anciens enseignants parmi ces quinze membres. «SBOE Members », Texas Education Agency, http://tea.texas.gov/index4.aspx?id=2147506719 (consulté le 25 fév. 2015).

23 «Le Travail éditorial», Savoir Livre, http://www.savoirlivre.com/edition-manuel/travaileditorial.php (consulté le 25 février 2015).
} 
Rachel Hutchins, "Construction et négociation de l'identité nationale. Une étude comparatiste des programmes et des manuels scolaires d'histoire pour le primaire en France et aux États-Unis », Histoire@Politique, n ${ }^{\circ} 26$, maiaoût 2015, www.histoire-politique.fr

d'origine africaine est toujours un sujet particulièrement brûlant ${ }^{24}$. Par ailleurs, les manuels étatsuniens les plus récents suivent les attaques du 11 septembre $2001^{25}$. À la lumière de ce contexte de remise en question de l'identité nationale en France et aux États-Unis, cet article cherchera à élucider les objectifs nationalistes (dans le sens défini plus haut) de l'enseignement en général et de l'enseignement de l'histoire en particulier dans les deux pays. Nous explorerons comment les réécritures de l'histoire scolaire reflètent les différentes conceptions de la nation et contribuent à la redéfinir et, par ce biais, la perpétuent. Nous examinerons la forme que prend le nationalisme dans les deux pays et les valeurs qu'il véhicule. À ces fins, nous considérerons dans un premier temps l'image de la nation dans les manuels scolaires, notamment la place des femmes et les représentations des différents groupes ethniques, ainsi que certains sujets sensibles relatifs à la discrimination et à l'oppression raciale ou sexiste, oubliés des récits historiques traditionnels. Sera ensuite examinée la place des héros nationaux dans ces manuels. Enfin, nous esquisserons une analyse des idéaux et des valeurs fondatrices exprimés par ces ouvrages ${ }^{26}$.

\section{Le(s) visage(s) de la nation}

Examiner les illustrations des manuels scolaires pour le primaire permet de discerner les sujets que les éditeurs souhaitent mettre en valeur. Ces images sont abondantes et ont à la fois une fonction pédagogique pour les jeunes élèves et servent - surtout aux États-Unis - à indiquer aux examinateurs qui parcourent ces livres lors des processus de sélection des manuels par l'État - ainsi que pour les écoles individuelles - quels sont les thèmes mis en avant ${ }^{27}$. Nous examinerons la place des femmes et des minorités ethniques dans ces illustrations afin de déterminer quelle image des membres de la nation s'en dégage. Ces illustrations seront appréhendées à la fois d'un point de vue quantitatif (combien d'images montrent des femmes? des minorités ethniques ?) et qualitatif (dans quels rôles sont montrées les populations nationales dans leur ensemble? quels rôles sont privilégiés pour les hommes? pour les femmes? pour les différents groupes ethniques?).

Les représentations de la femme traditionnellement limitées aux rôles de mère, d'épouse, d'allégorie... ont longtemps fait partie intégrante des symboles de la nation. Ainsi, le fait d'inclure des femmes en tant qu'actrices de l'histoire dans des rôles divers confronte le féminisme au nationalisme traditionnel, qui repose sur des distinctions rigides entre les rôles réservés aux hommes et aux femmes ${ }^{28}$. Depuis les années 1960 aux États-Unis, des enseignants, des chercheurs et des associations féministes prônent l'inclusion d'un plus grand nombre de femmes dans les manuels

24 Gérard Noiriel, À quoi sert..., op. cit. ; Elaine Thomas, Immigration, op. cit. ; « Définir les programmes : entretien avec Laurent Wirth », Le Débat, n 175, 2013, p. 23.

${ }^{25} \mathrm{La}$ liste complète des ouvrages scolaires se trouve dans la bibliographie.

${ }^{26}$ Edward Berenson, Vincent Duclert et Christophe Prochasson, The French Republic: History, Values, Debates, Ithaca, NY, Cornell University Press, 2011; Élise Marienstras, Les Mythes fondateurs de la nation américaine, Paris, François Maspero, 1976.

${ }^{27}$ Harriet Tyson-Bernstein et Arthur Woodward, « Nineteenth Century Policies for Twenty-First Century Practice», in P. Altbach et alii (eds.), Textbooks in American Society, op. cit., p. 96 ; C. Finn et D. Ravitch, The Mad, Mad World..., op. cit.

28 Voir Nira Yuval Davis, Gender \& Nation, Londres, SAGE, 1997; George Mosse, The Image of Man: The Creation of Modern Masculinity, Oxford, Oxford University Press, "Studies in the History of Sexuality", 1998. 
Rachel Hutchins, "Construction et négociation de l'identité nationale. Une étude comparatiste des programmes et des manuels scolaires d'histoire pour le primaire en France et aux États-Unis », Histoire@Politique, n ${ }^{\circ} 26$, maiaoût 2015, www.histoire-politique.fr

scolaires et réclament que les femmes soient montrées de telle sorte que l'image aille à l'encontre de stéréotypes sexistes. Par ailleurs, si on ne s'attend pas à atteindre la parité dans les manuels d'histoire, les éditeurs peuvent néanmoins inclure des femmes à travers l'histoire nationale dans des rôles variés. Or, comme le montre le tableau 1, on constate une baisse de la visibilité des femmes depuis le début des années 1980.

Tableau 1: Les femmes et les hommes dans les illustrations - États-Unis

\begin{tabular}{|r|l|l|}
\hline & $\begin{array}{l}\text { Pourcentage d'illustrations } \\
\text { comprenant des femmes }\end{array}$ & $\begin{array}{l}\text { Pourcentage d'illustrations comprenant } \\
\text { des hommes }\end{array}$ \\
\hline 1982 & $42 \%$ & $85 \%$ \\
\hline 1997 & $36 \%$ & $83 \%$ \\
\hline 2003 & $35 \%$ & $87 \%$ \\
\hline
\end{tabular}

Cette baisse est encore plus marquée si l'on ne considère que les illustrations du récit historique dans ces manuels, comme le montre le tableau 2.

Tableau 2 : Les femmes et les hommes dans les illustrations du récit historique - ÉtatsUnis

\begin{tabular}{|r|r|rr|}
\hline & $\begin{array}{l}\text { Illustrations historiques comprenant } \\
\text { des femmes }\end{array}$ & $\begin{array}{l}\text { Illustrations historiques comprenant } \\
\text { des hommes }\end{array}$ \\
\hline 1982 & $39 \%$ & $86 \%$ \\
\hline 1997 & $30 \%$ & $84 \%$ \\
\hline 2003 & $30 \%$ & $90 \%$ \\
\hline
\end{tabular}

Ce constat s'explique en grande partie par un déclin de l'histoire sociale dans ces manuels car les femmes sont souvent montrées dans des scènes de groupe ou de famille. Dans les manuels de 1982, $59 \%$ des illustrations du récit historique mettaient en scène uniquement des personnes anonymes. Ce chiffre baisse à $51 \%$ dans les ouvrages de 1997 et à $32 \%$ en 2003. Par ailleurs, le pourcentage d'images montrant des soldats augmente (passant de $19 \%$ en 1982 à $27 \%$ d'un nombre d'illustrations bien supérieur en 2003), réduisant ainsi davantage la visibilité des femmes et reléguant de plus en plus les hommes à des rôles traditionnels de défense dela patrie.

Cet éloignement de l'histoire sociale au profit de l'histoire militaire et politique est en accord avec les vœeux des organismes de la droite chrétienne qui sont actifs dans de nombreux États américains, et particulièrement au Texas. Ces groupes sont antiféministes et œuvrent pour le retour à des récits traditionnels en histoire ${ }^{29}$. Même

29 Plusieurs organismes issus de la droite chrétienne se mobilisent à travers les États-Unis afin d'influencer le contenu des programmes et des manuels scolaires, ainsi que les choix de manuels par les écoles et les enseignants. Ils sont particulièrement actifs en amont du processus de production des manuels au Texas, grâce au système employé par cet État. Ces groupes réclament généralement la mise en avant de la grandeur des Etats-Unis, le patriotisme (tels qu'ils le définissent), les héros traditionnels de la nation et de l'histoire politique et militaire. $\mathrm{Si}$, depuis les années 1990, ils acceptent que soient mentionnés certains événements ou périodes définis par l'injustice raciale (tels que l'esclavage ou la 
Rachel Hutchins, "Construction et négociation de l'identité nationale. Une étude comparatiste des programmes et des manuels scolaires d'histoire pour le primaire en France et aux États-Unis », Histoire@Politique, n² 26, maiaoût 2015, www.histoire-politique.fr

si les femmes ont une place moindre dans ces manuels, elles sont néanmoins représentées dans des rôles variés, y compris les femmes pendant la Révolution américaine (une image courante, qui figure ci-dessous - image 1-, fait état d'une femme remplaçant son mari au front), des femmes pilotes dans les années 1920, et les femmes au travail pendant la Seconde Guerre mondiale. Ces images sont d'ordre nationaliste, mettant en scène les femmes participant à la construction de la nation et de l'identité nationale. Il semblerait que les éditeurs des manuels tentent de concilier les demandes des conservateurs avec certaines considérations féministes, car s'ils mettent en valeur des femmes dans des rôles non traditionnels, ils mettent surtout l'accent sur une vision positive du pays.

Image 1: Héroïne de la Révolution américaine ${ }^{30}$. ๑ Droits réservés.

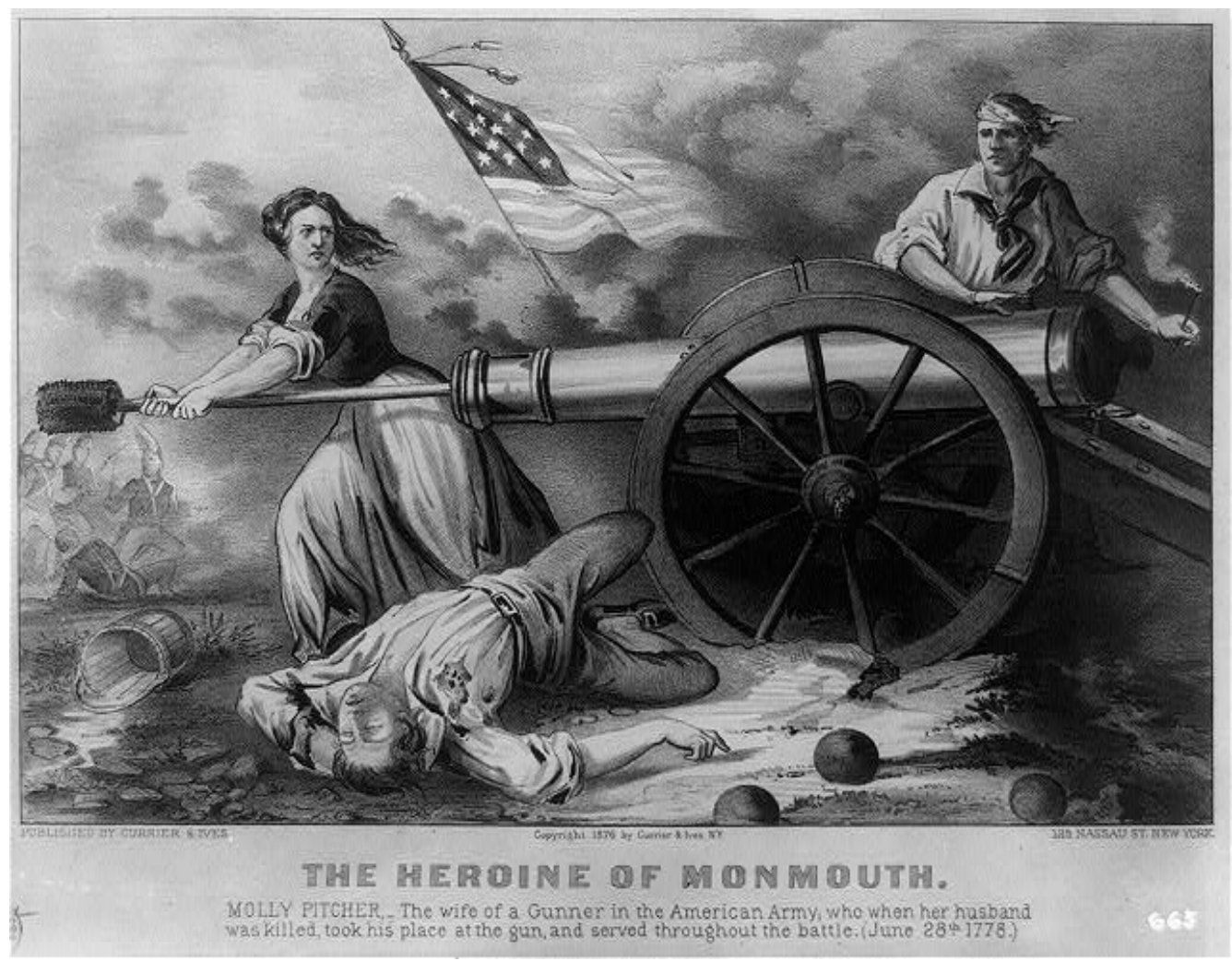

ségrégation légale), un grand nombre de ces partisans s'opposent en pratique à ce qu'ils perçoivent comme une insistance exagérée sur ces faits qui, selon eux, ternissent l'image qu'auront les enfants du pays. Pour une plus analyse plus fouillée de ces groupes, voir Rachel Hutchins, « Multiculturalism in American History Textbooks before and after 9/11 », dans Derek Rubin et J aap Verheul (eds.), American Multiculturalism After 9/11. Transatlantic Perspectives, Amsterdam, Amsterdam University Press, 2009.

30 Cette image ressemble à celles qui figurent dans de nombreux manuels étatsuniens. Mais les illustrations choisies par les éditeurs n'étant pas libres de droits, nous la substituons par celle-ci. Currier \& Ives, « The Heroine on Monmouth. Molly Pitcher ... June 28, 1778 », New York, Currier \& Ives, 1876. Disponible sur: Library of Congress, Prints and Photographs Online Collection, <http:// www.loc.gov/ pictures/item/2006691580/ > (consultéle 10 avril 2015). 
Rachel Hutchins, «Construction et négociation de l'identité nationale. Une étude comparatiste des programmes et des manuels scolaires d'histoire pour le primaire en France et aux États-Unis », Histoire@Politique, n² 26, maiaoût 2015, www.histoire-politique.fr

Dans les manuels français, on constate une plus grande inclusion de femmes après les années 1980, bien que leur visibilité n'évolue pas de manière linéaire, comme le montre le tableau 3.

Tableau 3 : Les femmes et les hommes dans les illustrations - France

\begin{tabular}{|r|r|lr|}
\hline & $\begin{array}{l}\text { Illustrations comprenant des } \\
\text { femmes }\end{array}$ & $\begin{array}{l}\text { Illustrations comprenant des } \\
\text { hommes }\end{array}$ \\
\hline $1985 \mathrm{CM}$ & $33 \%$ & $95 \%$ \\
\hline $1997 \mathrm{CM} 1$ & $34 \%$ & $95 \%$ \\
\hline $1997 \mathrm{CM} 2$ & $42 \%$ & $93 \%$ \\
\hline $2003 \mathrm{CM} 1$ & $37 \%$ & $93 \%$ \\
\hline $2003 \mathrm{CM} 2$ & $48 \%$ & $87 \%$ \\
\hline $2010 \mathrm{CM} 1$ & $34 \%$ & $95 \%$ \\
\hline $2010 \mathrm{CM} 2$ & $43 \%$ & $89 \%$ \\
\hline
\end{tabular}

Bien entendu, le pourcentage de femmes baisse lorsqu'on exclut les illustrations contemporaines concernant la géographie et l'éducation civique pour considérer uniquement les parties des ouvrages portant sur l'histoire, comme le montre le tableau 4.

Tableau 4 : Les femmes et les hommes dans les illustrations du récit historique - France

\begin{tabular}{|r|r|r|}
\hline & $\begin{array}{l}\text { Ilustrations historiques } \\
\text { comprenant des femmes }\end{array}$ & $\begin{array}{l}\text { Illustrations historiques } \\
\text { comprenant des hommes }\end{array}$ \\
\hline $1985 \mathrm{CM}$ & $29 \%$ & $96 \%$ \\
\hline $1997 \mathrm{CM} 1$ & $25 \%$ & $96 \%$ \\
\hline $1997 \mathrm{CM} 2$ & $39 \%$ & $93 \%$ \\
\hline $2003 \mathrm{CM} 1$ & $30 \%$ & $93 \%$ \\
\hline $2003 \mathrm{CM} 2$ & $41 \%$ & $87 \%$ \\
\hline $2010 \mathrm{CM} 1$ & $31 \%$ & $96 \%$ \\
\hline $2010 \mathrm{CM} 2$ & $38 \%$ & $90 \%$ \\
\hline
\end{tabular}

Le pourcentage d'hommes dans les illustrations reste très élevé car lorsque les femmes sont montrées, elles ont souvent des hommes à leur côté. Dans ce cas de figure, ces derniers tiennent la plupart du temps des rôles actifs dans les images, alors que les femmes sont reléguées au second plan ou sont passives. Par ailleurs, lorsqu'elles sont montrées seules, les figures féminines sont souvent (environ une fois sur deux en 1985 et une fois sur trois dans les manuels les plus récents) celles d'allégories ou d'œeuvres d'art. Si on enlevait ces derniers types d'images des chiffres totaux il ne resterait plus que $20 \%$ d'illustrations comportant des femmes dans les manuels les plus récents de CM1 (15\% si on enlevait J eanne d'Arc) et plus que $26 \%$ pour le CM2.

Si les femmes restent peu visibles et sont souvent montrées en tant qu'objets (que ce soit symbole national ou plutôt dans des œuvres d'art), les ouvrages français 
Rachel Hutchins, "Construction et négociation de l'identité nationale. Une étude comparatiste des programmes et des manuels scolaires d'histoire pour le primaire en France et aux États-Unis », Histoire@Politique, n² 26, maiaoût 2015, www.histoire-politique.fr

commencent néanmoins à adopter une approche critique de l'exclusion des femmes de la vie publique. Cette démarche est conforme aux instructions des programmes depuis 2002, qui exigent désormais qu'«à chaque fois que cela est possible, on souligne le rôle des femmes dans la vie publique, en s'interrogeant sur leur faible place ${ }^{31} \gg$.

Cette instruction fait partie d'un changement significatif dans les programmes. Les programmes précédents mentionnaient peu de femmes nommément (deux personnes à connaître sur les vingt nommées en 1995 sont des femmes) et n'attiraient pas l'attention sur l'histoire des femmes en France. Les documents de 2002 ajoutent quelques femmes supplémentaires à la liste de personnages à connaître, mentionnent les femmes de la Révolution, les femmes au travail et le droit de vote pour les femmes en 1944. Au-delà de ces quelques éléments supplémentaires de l'histoire des femmes, ces programmes innovent surtout en intégrant un regard critique concernant l'exclusion des femmes des récits d'histoire et de la vie publique dans le passé. Ce changement majeur reflète sans doute une tendance plus générale: Christine Bard affirme que l'année 1995 marque un tournant dans les études féministes en France et dans la place du féminisme dans la société française de manière générale. Bien que les études féministes existaient depuis les années 1970 en France, elles peinaient à se faire reconnaître. C'est à la suite de la conférence mondiale de Pékin sur l'égalité hommes-femmes et des manifestations en France en 1995 que le climat politique a changé. Par exemple, la loi sur la parité (2000), ainsi que d'autres mesures officielles pour réduire l'inégalité, datent de cette époque ${ }^{32}$. Concernant l'enseignement, en 2001, le gouvernement - plus précisément, la Direction de l'Enseignement supérieur du ministère de l'Éducation nationale et de la Recherche- cherche également à faire un état des lieux des études sur le genre en France ${ }^{33}$. De même, en 2003, le Conseil économique et social a commandé une étude intitulée «Quelle place pour les femmes dans l'histoire enseignée ${ }^{34}$ ? ». La plus grande sensibilité des instances publiques aux questions d'égalité homme-femme se traduit à la même époque dans les programmes d’histoire.

Tous les ouvrages récents attirent l'attention des élèves sur l'exclusion des femmes des droits républicains à des moments différents de l'histoire, et deux des trois manuels de CM2 les plus récents indiquent qu'il reste des progrès à faire pour atteindre l'égalité entre les hommes et les femmes (Hatier et Sedrap). Toutefois, un seul ouvrage adopte un regard critique sur l'image des femmes dans la publicité d'autrefois et, là encore, l'image ainsi remise en question concerne les relations au sein du couple ; aucun ouvrage ne prend en revanche de distance critique par rapport aux images plus sexualisées des femmes qui ne sont généralement pas commentées.

\footnotetext{
${ }^{31}$ Ministère de la J eunesse, de l'Éducation nationale et de la Recherche : Direction de l'enseignement scolaire ; « Histoire et géographie cycle des approfondissements (cycle 3) », coll. «École, Documents d'application des programmes », Paris, Centre national de documentation pédagogique, 2002, p. 9.

32 Christine Bard, «J Jalons pour une histoire des études féministes en France (1970-2002) », Nouvelles questions féministes, tome $22, \mathrm{n}^{\circ} 1,2003, \mathrm{p} .24$.

33 Muriel Andriocci et alii, « Premier recensement national des enseignements et des recherches sur le genre en France», Paris, Association nationale des études féministes, 2003, http:// www.anef.org/ ?p=308 (consulté le 25 février 2015).

34 Annette Wieviorka, «Quelle place pour les femmes dans l'histoire enseignée? », Conseil économique et social, 2004, http:// www.haut-conseil-egalite.gouv.fr/IMG/pdf/ 04022705pdf-b193.pdf (consulté le 25 février 2015).
} 


\section{H@P}

Rachel Hutchins, "Construction et négociation de l'identité nationale. Une étude comparatiste des programmes et des manuels scolaires d'histoire pour le primaire en France et aux États-Unis », Histoire@Politique, n² 26, maiaoût 2015, www.histoire-politique.fr

On peut ainsi encore trouver l'affiche publicitaire ci-dessous (image 2) dans le manuel de CM2 d'Hatier datant de 2004.

\section{Image 2 : Publicité Rayon d'or ${ }^{35}$. ๑ Droits réservés.}

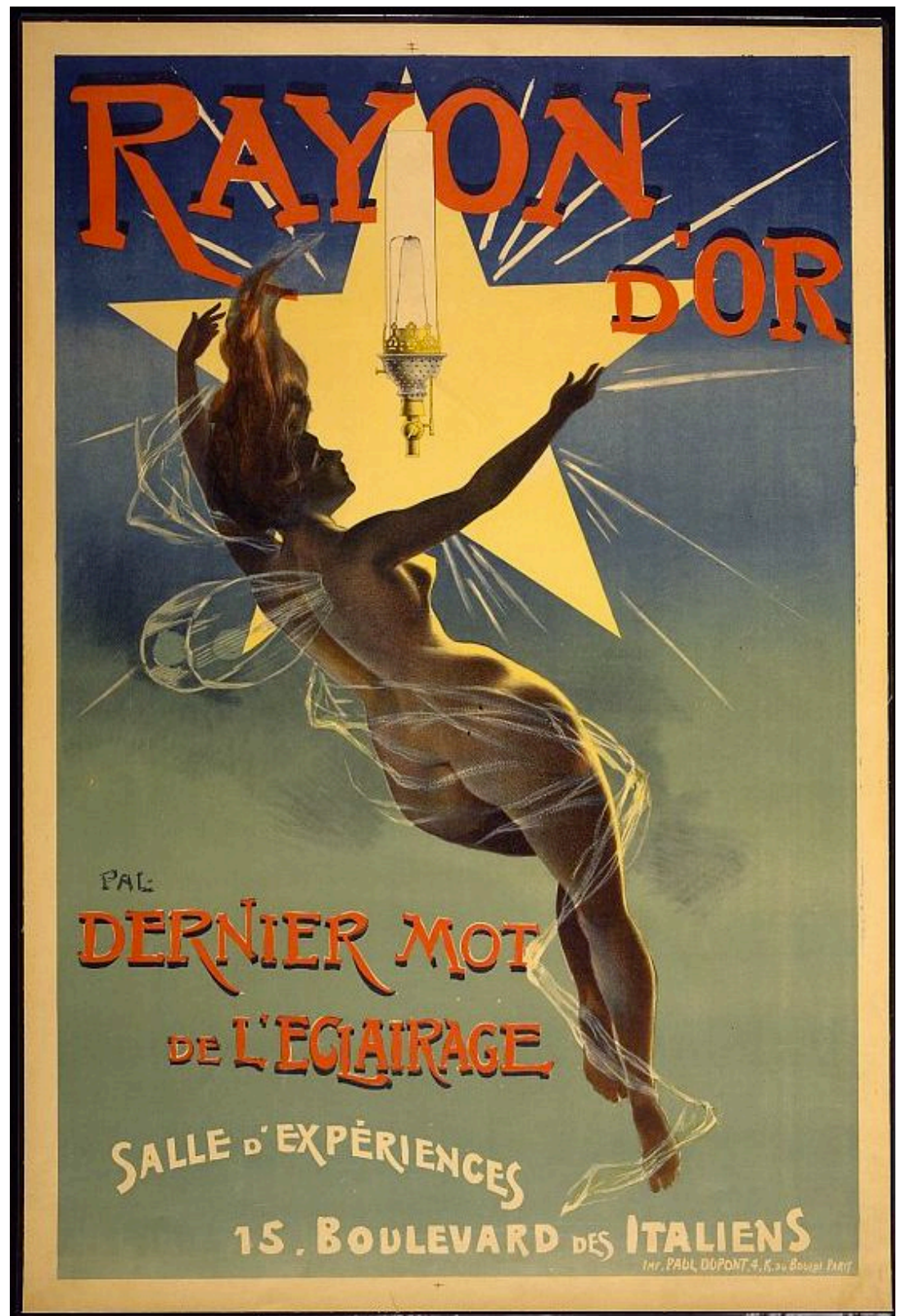

35 Document d'origine: Jean de Paleologue, «Rayon d'or, dernier mot de l'éclairage», Paris, Paul Dupont, 1895. Disponible sur: Library of Congress, Prints and Photographs Online Catalog, http:// www.loc.gov/ pictures/item/2004675013/ (consultéle 2 avril 2015). 
Rachel Hutchins, "Construction et négociation de l'identité nationale. Une étude comparatiste des programmes et des manuels scolaires d'histoire pour le primaire en France et aux États-Unis », Histoire@Politique, n² 26, maiaoût 2015, www.histoire-politique.fr

Globalement néanmoins, les manuels français semblent évoluer, poussés par les programmes, vers une position plus critique du sexisme. En cela, ces ouvrages diffèrent des manuels américains. Si ces derniers montrent des femmes dans des rôles plus variés, leur discours sur l'égalité entre les sexes s'est fait plus conservateur au cours des dernières années. Dans les manuels de 1982, par exemple, les auteurs expliquent que l'égalité a bien avancé, mais qu'il reste encore des progrès à faire pour que les femmes soient payées autant que les hommes ou puissent exercer toutes les professions. Les ouvrages les plus récents, en revanche, indiquent que l'égalité est désormais acquise, citant comme preuve l'accession de femmes à la Cour Suprême ou à d'autres postes de haute responsabilité politique.

La représentation des minorités ethniques, en particulier celle traitant de la discrimination à leur égard, révèlent des évolutions parallèles à celles concernant le féminisme. Connaissant la politique différente en France et aux États-Unis en ce qui concerne les données démographiques, on s'attend à ce que les programmes et les manuels scolaires abordent ces sujets d'une toute autre manière ${ }^{36}$. Néanmoins, la comparaison est révélatrice de tendances générales.

Comme nous l'avons vu plus haut, les programmes français postérieurs à 1985 mettent davantage l'accent sur la culture commune française. À l'époque où plusieurs historiens revendiquaient un certain pluralisme, les programmes de 1985 suggéraient quelques ouvertures sur d'autres pays en fonction des origines des élèves de la classe, mais ce n'est plus le cas dans les programmes suivants. Ils affirment désormais que l'enseignement a comme objectif de transmettre une culture partagée, dans laquelle l'histoire, la géographie et l'éducation civique tiennent un rôle important. Par exemple, concernant les listes d'événements et de personnages à connaître, le programme de CM2 d'histoire en 2008 affirme: «Jalons de l'histoire nationale, ils forment la base d'une culture commune ${ }^{37}$. » Ces programmes prônent également la tolérance et le respect de la diversité, citant comme exemple les langues régionales françaises. Toutefois, ils mettent avant tout l'accent sur la culture et l'appartenance nationales.

Les programmes américains, quant à eux, sont pluralistes. Ils donnent l'image d'une culture américaine unie par des valeurs civiques communes, dont la célébration de la diversité culturelle des individus et des groupes. Contrairement aux programmes français, ils évoquent explicitement les minorités ethniques. Par exemple, le programme texan le plus récent (pour lequel il n'existe pas encore de manuels) reprend une idée présente tout au long de la période étudiée en exigeant que : «Les élèves décrivent l'impact culturel de divers groupes raciaux, ethniques et religieux dans la nation et identifient ce qu'ont accompli des individus remarquables dans les sciences et la technologie ${ }^{38}$. »La représentation des minorités ethniques dans les manuels français est en revanche relativement faible. Le tableau 5 indique les

\footnotetext{
36 À l'inverse de la politique française qui interdit que les données officielles prennent en compte l'appartenance ethnique des individus, le recensement étatsunien demande que l'on indique ses origines « raciales » et ethniques. Si les Français considèrent que la catégorisation ethnique des citoyens risque de perpétuer ou de créer des divisions et des appartenances aux groupes sous-nationaux, les Américains considèrent que pour combattre les injustices racistes du passé, il faut pouvoir quantifier les inégalités actuelles.

37 « Horaires et programmes...2008 », op. cit., p. 25.

38 « Chapter 113: Texas Essential Knowledge and Skills for Social Studies », Texas Essential Knowledge and Skills, Texas Education Agency, 2010, http://ritter.tea.state.tx.us/rules/tac/ chapter113/index.html (consulté le 5 nov. 2013), §113.7.a.1.
} 
Rachel Hutchins, «Construction et négociation de l'identité nationale. Une étude comparatiste des programmes et des manuels scolaires d'histoire pour le primaire en France et aux États-Unis », Histoire@Politique, n ${ }^{\circ} 26$, maiaoût 2015, www.histoire-politique.fr

pourcentages d'illustrations dans les manuels français montrant uniquement des personnes « blanches », ainsi que celles montrant des personnes de couleur.

Tableau 5 : Groupes ethniques dans les illustrations - Manuels français

\begin{tabular}{|r|r|r|r|}
\hline & $\begin{array}{r}\text { «lancs } \\
\text { seuls }\end{array}$ & $\begin{array}{r}\text { Inclut minorité } \\
\text { ethnique }\end{array}$ & $\begin{array}{r}\text { Nombre d'illustrations de } \\
\text { personnes en moyenne/ manuel }\end{array}$ \\
\hline $1985 \mathrm{CM}$ & $92 \%$ & $9 \%$ & 226 \\
\hline $1995 \mathrm{CM} 1$ & $93 \%$ & $7 \%$ & 123 \\
\hline $1995 \mathrm{CM} 2$ & $88 \%$ & $12 \%$ & 151 \\
\hline $2002 \mathrm{CM} 1$ & $88 \%$ & $12 \%$ & 93 \\
\hline $2002 \mathrm{CM} 2$ & $78 \%$ & $22 \%$ & 124 \\
\hline $2010 \mathrm{CM} 1$ & $90 \%$ & $10 \%$ & 110 \\
\hline $2010 \mathrm{CM} 2$ & $80 \%$ & $20 \%$ & 138 \\
\hline
\end{tabular}

Dans l'ensemble, les manuels les plus récents donnent une image plus diverse de la nation que ne le faisaient les éditions des années 1980, bien qu'aucun consensus ne se dégage dans le traitement de cette question. Aujourd'hui, tous les ouvrages consacrent davantage de place aux départements et aux territoires d'outre-mer que ne le faisaient leurs prédécesseurs. Mais, dans la plupart d'entre eux, ces régions ne sont pas pleinement incorporées dans l'entité «France». Pour les ouvrages de Sedrap et de Nathan, « France » renvoie généralement à l'hexagone. L'outre-mer fait l'objet de développements spécifiques (et séparés) dans les chapitres sur « La France dans le monde», traitant ainsi ces régions dans les mêmes chapitres que les pays francophones. De même, dans les manuels d'histoire et de géographie, peu d'individus issus des minorités ethniques sont mis en images pour illustrer la France métropolitaine. La grande majorité des ouvrages ne contient aucune image accompagnant le récit historique mettant en scène des personnes issues de minorités ethniques en France métropolitaine (un seul le fait, et il s'agit d'un serviteur noir non mentionné, figurant en marge de l'action d'un tableau). Dans les leçons de géographie, parmi les ouvrages de 2010, en moyenne une image par manuel montre une personne de couleur en France métropolitaine. Notons toutefois que les rares ouvrages d'éducation civique donnent une image bien plus diversifiée de la France : par exemple, le manuel d'instruction civique publié en 2008 par Hatier inclut des personnes de couleur dans $38 \%$ des illustrations. Notons également qu'aucune personne portant des signes visibles d'appartenance à la confession musulmane ou identifiée par le texte comme tel n'apparaît dans ces ouvrages. Cela confirme l'idée selon laquelle ces manuels présentent une vision assimilationniste du pays, et se font le reflet de la loi de 2004, qui interdit le port de tout signe d'appartenance religieuse à l'école, contrairement, par exemple, aux manuels américains, où le hijab sert à distinguer des Américaines de confession musulmane, confirmant l'accent mis par ces manuels sur le pluralisme culturel 39 .

$\mathrm{Si}$ les programmes d'éducation civique exigent que les élèves apprennent à rejeter la discrimination, les programmes d'histoire, en revanche, n'évoquent pas le

\footnotetext{
39 McGraw-Hill, 2003, p. 599 ; 603.
} 
Rachel Hutchins, "Construction et négociation de l'identité nationale. Une étude comparatiste des programmes et des manuels scolaires d'histoire pour le primaire en France et aux États-Unis », Histoire@Politique, n² 26, maiaoût 2015, www.histoire-politique.fr

phénomène de la discrimination à l'époque contemporaine, alors que le fait de confronter les élèves à l'injustice constitue un moyen puissant de la combattre ${ }^{40}$. Aussi la discrimination n'est-elle que rarement évoquée dans les manuels d’histoire. Parmi les manuels les plus récents, seul Sedrap mentionne la discrimination à l'égard des immigrés récents, alors qu'elle était plus souvent évoquée dans les manuels antérieurs, surtout vers la fin des années 1990.

S'ils ne traitent pas le sujet de la situation des immigrés depuis la deuxième moitié du $\mathrm{XX}^{\mathrm{e}}$ siècle, des changements significatifs se sont opérés au cours des trente dernières années dans le traitement de l'oppression raciale à l'époque moderne et au XIX siècle. Par exemple, concernant la présentation de la colonisation et de la décolonisation françaises, bien qu'ils abordent encore ces sujets de manière différente, tous les manuels évitent l'approche traditionnaliste encore présente dans les années 1980, qui consistait à présenter les conquêtes coloniales françaises comme une source de fierté et de force nationale. Concernant la décolonisation, les manuels récents montrent davantage le rôle joué par les résistants dans les pays colonisés, restituant ainsi leur place en tant qu'acteurs et agents de leur histoire. Toutefois, on constate que ces ouvrages ont encore tendance à présenter la décolonisation comme un signe de progrès français (impression qui est renforcée par l'accent mis sur les bonnes relations au sein de la « Francophonie»). De même, concernant l'esclavage, les manuels soulignent davantage le rôle joué par la France et la présence d'esclaves dans les territoires français d'outre-mer. Mais la plupart d'entre eux présentent l'abolition de l'esclavage d'un point de vue eurocentriste et comme un triomphe de la République, insistant sur l'action de Victor Schoelcher et de l'État, et passant sous silence le rôle majeur joués par certains esclaves comme Toussaint Louverture et Louis Delgrès, seulement évoqué dans l'ouvrage de Sedrap ${ }^{41}$.

Sur certains sujets, en effet, les manuels français adoptent un point de vue eurocentriste, présentant les événements selon la lecture traditionnelle européenne, sans prendre en compte les points de vue des autres groupes ethniques. Cette approche domine la présentation des Croisades et de ce que les manuels appellent la « découverte » de l'Amérique et de l'Afrique, ignorant ainsi que ces continents étaient déjà connus par ceux qui y habitaient. Globalement, les manuels français accordent plus de place à l'histoire des minorités ethniques dans le passé national et mondial, mais ces groupes restent encore en marge dans la plupart des ouvrages et le point de vue reste le plus souvent celui des Français d'origine européenne.

Alors que les manuels français deviennent plus critiques vis-à-vis des injustices commises par la majorité ethnique nationale dans le passé, la trajectoire des manuels américains est beaucoup moins linéaire. Au cours des années 1970, les maisons d'édition ont mis en place des systèmes de quotas qui déterminent le pourcentage minimal d'illustrations devant inclure des minorités ethniques. Ces pourcentages n’ont guère évolué au cours de la période étudiée ici, comme le montre le tableau 6.

\footnotetext{
40 Zoë Burkholder, Color in the Classroom : How American Schools Taught Race, 1900-1954, Oxford ; New York, Oxford University Press, 2011.

41 Sedrap, CM2, 2010, p. 21; 115.
} 
Rachel Hutchins, «Construction et négociation de l'identité nationale. Une étude comparatiste des programmes et des manuels scolaires d'histoire pour le primaire en France et aux États-Unis », Histoire@Politique, n ${ }^{\circ} 26$, maiaoût 2015, www.histoire-politique.fr

Tableau 6: Groupes ethniques dans les illustrations - Manuels américains

\begin{tabular}{|r|r|r|r|}
\hline & “Blancs” seuls & $\begin{array}{r}\text { Inclut minorité } \\
\text { ethnique }\end{array}$ & $\begin{array}{r}\text { Nombre d'illustrations } \\
\text { de personnes en } \\
\text { moyenne/manuel }\end{array}$ \\
\hline 1982 & $58,3 \%$ & $41,7 \%$ & 117 \\
\hline 1997 & $61,2 \%$ & $38,8 \%$ & 374 \\
\hline 2003 & $59,5 \%$ & $40,5 \%$ & 427 \\
\hline
\end{tabular}

Toutefois, comme le nombre d'images par manuel augmente de façon considérable (passant d'environ 117 en moyenne par manuel en 1982 à 427 en 2003), la nature de ces images évolue sans avoir d'effet sur les pourcentages. Contrairement aux ouvrages français, plusieurs personnes de couleur figurent parmi les personnages le plus souvent représentés en images dans les manuels américains. Parmi ceux-ci, certains sont chers aux groupes multiculturalistes (tels que Martin Luther King, Jr., Frederick Douglass, Harriet Tubman...). Mais de manière générale, les acteurs les plus radicaux - dont les groupes multiculturalistes réclament l'inclusion dans les manuels d'histoire - sont absents, et certains personnages bien visibles dans les manuels sont considérés par des multiculturalistes comme perpétuant des stéréotypes ou des mythes nuisibles à l'égard des groupes en question : par exemple, les Amérindiennes Sacajawea et Pocahontas, qui furent d'une grande aide aux colons $^{42}$. Le même procédé caractérise le choix des femmes, car généralement, les féministes contemporaines telles que Gloria Steinem ou Betty Friedan ne figurent pas dans les ouvrages les plus récents. Ainsi, bien que les Afro-américains et les Amérindiens occupent une place importante dans ces ouvrages, il s'agit d'une sorte de compromis. On met en valeur la diversité ethnique de la nation tout en minimisant l'impression de conflit et d'inégalité persistante à l'époque contemporaine, est largement minimisée.

La persistance du point de vue conservateur sur les injustices raciales dans l'histoire américaine est également évidente dans l'évolution des sujets traités par les manuels. Conformément aux travaux d'historiens et aux souhaits des groupes multiculturalistes, les manuels de 1997 incluent souvent des récits et des témoignages montrant la souffrance des victimes de l'oppression raciste, que cela concerne des esclaves ou des Américains d'origine japonaise internés dans des camps lors de la Deuxième Guerre mondiale. Ces ouvrages accordent plus de place à ces sujets que ne le faisaient les manuels de 1982. En revanche, les manuels de 2003 édulcorent leurs propos. Sans toutefois les enlever des récits, ils insistent beaucoup moins sur l'injustice et la souffrance. Par exemple, lorsqu'on compare les chapitres sur l'esclavage dans les éditions successives d'un même ouvrage paru chez McGraw-Hill, on constate que trois textes, écrits par deux esclaves et par un observateur abolitionniste évoquant la séparation des enfants esclaves de leur mère et la cruauté subie quotidiennement sur une plantation ${ }^{43}$, sont absents de celui de 2003. Ils étaient pourtant présents dans le manuel de 1997...

42 Robert Moore, Stereotypes, Distortions, and Omissions in U.S. History Textbooks, New York, Council of Interracial Books for Children, 1977, p. 106.

43 McGraw Hill 1997, p. 236, 444, 445. 
Rachel Hutchins, "Construction et négociation de l'identité nationale. Une étude comparatiste des programmes et des manuels scolaires d'histoire pour le primaire en France et aux États-Unis », Histoire@Politique, n ${ }^{\circ} 26$, maiaoût 2015, www.histoire-politique.fr

\section{Les héros nationaux}

La représentation des héros constitue encore une différence significative entre les manuels français et américains. Dans les deux pays, les années 1960 ont vu de nombreux historiens s'éloigner du récit historique centré sur les « grands hommes ». Toutefois, en France, depuis le retour, dans les années 1980, de l'histoire comme discipline scolaire distincte après les années de «l'éveil », un grand nombre d'historiens et d'hommes politiques ont réclamé un retour partiel à l'histoire événementielle (sans pour autant rejeter complètement l'histoire sociale ou les nouvelles démarches épistémologiques). Ainsi, un nombre accru de personnages à connaître est mentionné dans les programmes successifs. Le programme de 1985 cite uniquement trois personnages: il indique que les élèves doivent étudier «la monarchie et la formation de l'État (de Philippe Auguste à Louis XI) », ainsi que « la France et l’Europe au temps de Napoléon 44 ». À partir de 1995, les programmes énumèrent des listes de figures historiques que les élèves de troisième cycle du primaire doivent connaître, et ce nombre augmente, passant de vingt personnes dans le programme de 1995 à trente-cinq dans le programme de 2002 et quarante dans celui de 2008.

Même en incluant bon nombre de figures connues, la majorité des illustrations dans les manuels français mettent en scène des inconnus, accordant ainsi une place considérable à l'histoire sociale. En revanche, dans les ouvrages américains, le nombre et le pourcentage d'illustrations montrant des personnes connues ont augmenté de manière considérable depuis 1982. Parmi les 117 illustrations de personnes en moyenne par manuel en 1982, plus de la moitié montraient des anonymes. Mais dans les ouvrages de 2003, seul un tiers de ces 427 illustrations en faisait autant. Ce changement reflète pour partie un certain consensus : bien que les historiens mettent davantage l'accent sur l'histoire sociale, un grand nombre d'acteurs impliqués dans le processus de rédaction des programmes et des manuels scolaires américains réclament la présence de héros qui leur sont chers. Pour certains multiculturalistes, cela implique l'inclusion d'un plus grand nombre de femmes et de personnes issues des minorités ethniques. Pour les conservateurs, la présence des héros traditionnels est essentielle.

Quant aux ouvrages français, le retour à certains éléments de l'histoire événementielle ne signifie pas l'abandon de toutes les évolutions des années 1960 et 1970. Tout en présentant les personnages préconisés par les programmes, les manuels s'éloignent progressivement de l'hagiographie ${ }^{45}$. Si, dans les années 1980, on trouvait encore parfois des présentations glorifiant Napoléon I ${ }^{\mathrm{er}}$ ou Charles de Gaulle. Aujourd'hui, de manière générale, les manuels scolaires ne font pas de commentaire élogieux sur le caractère des personnages et n'en livrent pas une image idéalisée. Habituellement, ils accordent un maximum de deux pages à chaque personnage. Les figures les plus souvent représentées en images (Charles de Gaulle et J eanne d'Arc) apparaissent en moyenne environ cinq fois par livre. Dans l'ensemble, même si certains personnages sont présentés de manière favorable (de Gaulle, Henri IV, Victor Hugo, Marie Curie, etc.), la place qui leur est accordée semble être déterminée

\footnotetext{
${ }^{44}$ Horaires..., op. cit., arrêté du 23 avril 1985, Histoire et géographie, « Programmes », RLR 514-4, p. 19. 45 Cette conclusion rejoigne celles d'autres chercheurs. Cf. G. Bozec, Les héritiers..., op. cit., p. 198-199. Hanna Schissler et Yasemin Nuhoglu Soysal, The Nation, Europe, and the World: Textbooks and Curricula in Transition, New York; Oxford, Berghahn Books, 2005, p. 13-34.
} 
Rachel Hutchins, "Construction et négociation de l'identité nationale. Une étude comparatiste des programmes et des manuels scolaires d'histoire pour le primaire en France et aux États-Unis », Histoire@Politique, n ${ }^{\circ} 26$, maiaoût 2015, www.histoire-politique.fr

par l’ampleur de leur implication dans les événements ou la société de leur époque. Il existe néanmoins une exception majeure à ce constat : J eanne d'Arc. Après Charles deGaulle, elle est le personnage le plus visible dans les ouvrages. Les autres personnages figurant dans au moins trois illustrations en moyenne dans les ouvrages les plus récents (de Gaulle, Louis XIV, Napoléon Ier, Henri IV et Louis IX) furent tous des dirigeants politiques qui ont joué un rôle important dans l'histoire française et qui ont façonné les institutions de l'État. Les manuels indiquent alors que le rôle joué par J eanne d'Arc était d'une importance semblable. L'accent mis sur cette dernière s'explique peut-être en partie par les instructions des documents d'accompagnent (en vigueur pour les programmes de 2002 et de 2008) :

« La guerre de Cent Ans, avec son héroïne J eanne d'Arc, fait naître une première forme de conscience nationale, facilitée aussi par l'émergence de la langue française ${ }^{46}$. »

De même, quoique dans une moindre mesure, les manuels récents transmettent une vision positive de Louis IX, évoquant surtout la légende du roi qui dispensait la justice assis sous un chêne dans le bois de Vincennes. Dans ces cas, les manuels visent à perpétuer la mémoire nationale. Toutefois, dans l'ensemble, ils adoptent une approche des personnages historiques compatible avec l'historiographie savante.

$\mathrm{Si}$ les manuels américains ont ajouté un certain nombre de femmes et de minorités ethniques au panthéon national, conformément aux souhaits des multiculturalistes et conformément à l'historiographie savante qui prête davantage d'attention à ces groupes depuis les années 1960, ils multiplient aussi les images des figures phares de l'histoire nationale, et plus particulièrement de celles liées aux fondements de l'État. L'exemple le plus frappant de ce changement concerne George Washington, commandeur des forces américaines pendant la Révolution, premier président et premier héros national. La visibilité de Washington s'accroît de façon remarquable depuis 1982. Il figure en moyenne 4,8 fois par manuel en 1982, 12,8 fois en 1997 et 17,7 fois en 2003. Souvent, ces manuels reprennent plusieurs fois une même image, soulignant ainsi son importance. En présentant Washington, les images comme le texte mettent l'accent sur ses qualités de chef, son courage, son habilité militaire, son humilité, sa dévotion, son sens du devoir et du sacrifice, sa persévérance face aux obstacles et le respect qu'il inspira à ses soldats, aux chefs de la Révolution et au peuple américain.

Si Washington est pour la plupart des Américains un personnage majeur et digne d'honneur, il existe néanmoins une différence entre l'approche souhaitée par les multiculturalistes et les conservateurs concernant sa relation à l'esclavage. Pour les premiers, il faudrait évoquer le fait que le premier président, ainsi que de nombreux autres personnages importants de l'époque, possédaient des esclaves. Pour la droite chrétienne, l'image des «pères fondateurs » doit être protégée, afin de promouvoir l'amour du pays. Les manuels scolaires semblent avoir accepté cette dernière approche. Si en 1997 deux ouvrages sur quatre évoquaient le fait que Washington avait des esclaves, deux manuels sur trois de 2003 omettent ce fait et le troisième donne l'impression erronée que Washington avait libéré ses esclaves après la Révolution américaine.

${ }^{46}$ Ministère de la J eunesse..., « Histoire et géographie... », op. cit. p. 12. 
Rachel Hutchins, "Construction et négociation de l'identité nationale. Une étude comparatiste des programmes et des manuels scolaires d'histoire pour le primaire en France et aux États-Unis », Histoire@Politique, n ${ }^{\circ} 26$, maiaoût 2015, www.histoire-politique.fr

\section{La justice sociale}

Ce contraste entre une approche plus en adéquation avec l'historiographie savante dans les manuels français et une approche plus patriotique dans les ouvrages américains est encore plus frappant dans le traitement des valeurs de justice sociale, de solidarité et d'égalité. Dans les manuels français, le concept des classes sociales est un thème récurrent. La question des relations entre les classes socio-économiques joue un rôle majeur dans la présentation du Moyen Âge, des idées des Lumières, de la Révolution française, de la révolution industrielle et de l'époque contemporaine. Dans les manuels américains, les inégalités socio-économiques tiennent une place bien plus limitée et surtout semblent être reléguées dans un passé révolu. Cette situation a évolué de manière assez nette depuis les années 1980. À l'époque, les manuels scolaires américains évoquaient la persistance de la pauvreté dans les années 1960 malgré la prospérité générale du pays. Ces ouvrages faisaient mention de la «Guerre contre la pauvreté » du président Lyndon J ohnson. Même si la plupart d'entre eux n'évoquaient plus ce sujet après les années 1960, certains indiquaient que c'était encore un problème majeur dans la société américaine. Par exemple, l'ouvrage publié par Follett décrit clairement la pauvreté, soulignant le lien pouvant exister avec la discrimination. Il réclame même explicitement la réforme du système pour remédier à ces iniquités :

\footnotetext{
«Bien que les États-Unis aient une bonne qualité de vie, ils ont aussi de nombreuses personnes qui vivent dans la pauvreté. Elles vivent dans des quartiers pauvres des villes et dans des taudis à la campagne. Elles ont peu d'instruction, peu de nourriture et de vêtements, et peu d'espoir. Beaucoup sont au chômage et beaucoup sont obligées de vivre de l'argent du gouvernement. Certaines sont issues des minorités ethniques mais la majorité est blanche. Nous devons travailler sur notre système pour nous assurer que tous ont les mêmes chances.

«[...] Si on manque d'instruction et de qualifications et on fait parfois l'objet de discrimination, trouver un travail peut être difficile et décourageant ${ }^{47}$. »
}

Après la publication de ce manuel, les références à la pauvreté persistante aux ÉtatsUnis après la Deuxième Guerre mondiale disparaissent progressivement. Parmi les manuels de 2003, deux sur trois ne mentionnent pas les programmes de J ohnson et donnent ainsi l'impression que la pauvreté n'est plus un problème dans la société américaine. Le troisième ouvrage n'évoque la pauvreté actuelle que de manière indirecte et très rapide, en suggérant dans une question en fin de chapitre que l'élève imagine un programme semblable à ceux de Franklin Delano Roosevelt ou de Lyndon J ohnson, en en expliquant l'utilité.

Les manuels français, en revanche, mettent l'accent, en histoire comme en géographie sur les inégalités socio-économiques persistantes dans la société française et au niveau mondial. Parfois, ils attribuent explicitement ces inégalités et le ralentissement de l'économie en France et en Europe à la mondialisation. Par exemple, Sedrap présenteles années 1990 en ces termes :

"C'est aussi durant ces années que les échanges commerciaux se mondialisent, que la concurrence s'installe entre tous les pays du monde et que beaucoup d'usines sont fermées en France et en Europe et délocalisées dans les pays où la main-d'œuvre coûte moins cher.

${ }^{47}$ Follett 1980, p. 350-351. 
Rachel Hutchins, "Construction et négociation de l'identité nationale. Une étude comparatiste des programmes et des manuels scolaires d'histoire pour le primaire en France et aux États-Unis », Histoire@Politique, n ${ }^{\circ} 26$, maiaoût 2015, www.histoire-politique.fr

« Comme partout en Europe, la misère se répand en France, alors qu'un petit nombre de Français deviennent de plus en plus riches.

« Tout cela est amplifié (aggravé) suite aux énormes dégâts causés par la grave crise financière mondiale de septembre $2008^{48}$. »

Le plus souvent, la présentation de la mondialisation est plus subtile que dans cet extrait, mais néanmoins, en dénonçant les inégalités à l'échelle mondiale, les manuels critiquent, au moins de manière implicite, l'exploitation des pays pauvres par les pays riches.

Ces ouvrages soulignent l'importance de la solidarité à la fois dans la politique de l'État et dans les actions des individus. Ils visent ainsi à transmettre un engagement pour la justice sociale. Ceci reste vrai dans tous les manuels français de la présente étude, quel que soit le programme qu'ils suivent, quel que soit le pouvoir politique à l'origine des programmes. Peut-être faut-il y voir le reflet des orientations politiques de la majorité du corps enseignant et des chercheurs universitaires. Toutefois, ces valeurs semblent être assez consensuelles, dans la mesure où une grande majorité des Français se reconnaît dans ces idéaux, comme le montrent des sondages tels que l'International Social Survey Programme (ISSP) ${ }^{49}$. Souvent, les manuels associent cet engagement aux valeurs de la nation française, indiquant ainsi que les Français peuvent en être fiers - ce lien est encore plus explicite et souligné dans les manuels d'éducation civique. Ce thème apparaît dans les parties « histoire » des manuels lorsqu'ils évoquent la société française et le monde depuis la crise de 1973. L'ouvrage le plus récent de Nathan par exemple ouvre une partie intitulée "L'aide aux plus démunis » par un logo indiquant « Instruction civique », sur lequel figure un drapeau français. Ainsi, cet ouvrage indique que les actions pour promouvoir la solidarité relèvent du devoir citoyen. Les autres ouvrages étudiés ici (Hatier et Sedrap) évoquent de manière encore plus détaillée le sujet de l'égalité en France et à l'échelle de la planète dans les parties sur la géographie. Tous les trois (comme leurs prédécesseurs) indiquent que la France se situe parmi les pays riches, mais que des inégalités persistent également au sein de la société française. En tant que pays privilégié, selon ces manuels, la France (son gouvernement et ses citoyens) a le devoir d'aider les pays plus démunis.

Il convient de noter que la critique de la mondialisation semble aller de pair avec une certaine opposition implicite à l'américanisation ${ }^{50}$. Cet anti-américanisme se traduit

\footnotetext{
48 Sedrap CM2 2011, p. 76.

49 Le sondage de l'International Social Survey Programme de 2009 sur les inégalités sociales démontre par plusieurs questions qu'il existe un consensus différent en France et aux États-Unis concernant le rôle du gouvernement dans la redistribution des biens. Par exemple, en réponse à l'assertion « Le gouvernement a la responsabilité de réduire les différences entre les personnes percevant des revenus élevés et celles percevant des revenus faibles », 50,6\% des Français étaient « fortement d'accord » et 26,6\% étaient «d'accord»; par contraste, seulement 7,9\% des Américains étaient «fortement d'accord » et 24,7\% étaient « d'accord ». ISSP Research Group (2009), International Social Survey Programme: Social Inequality IV - ISSP 2009, GESIS Data Archive, Cologne, ZA4850 Data file Version 2.0 .0 doi:

10.4232/1.10079, http:// zacat.gesis.org/ webview/ index.jsp?object=http:// zacat.gesis.org/ obj/ fStudy/ ZA5400 (consulté le 20 mars 2014).

50 Concernant «l'américanisation» du monde, voir par exemple: Jean-Marie Guéhenno, « Américanisation du monde ou mondialisation de l’Amérique? », Politique étrangère, tome 64, $\mathrm{n}^{\circ} 1$, 1999, p. 7-20. Concernant l'anti-américanisme, voir par exemple: Sophie Meunier, «The Distinctiveness of French Anti-Americanism », dans Peter J. Katzenstein, Robert O. Keohane (eds.), Anti-Americanisms in World Politics, Ithaca, NY, Cornell University Press, 2006, p. 129-156.
} 
Rachel Hutchins, "Construction et négociation de l'identité nationale. Une étude comparatiste des programmes et des manuels scolaires d'histoire pour le primaire en France et aux États-Unis », Histoire@Politique, n ${ }^{\circ} 26$, maiaoût 2015, www.histoire-politique.fr

par l'accent mis dans plusieurs ouvrages sur l'importance de défendre la langue française face à la domination de l'anglais ${ }^{51}$. Par ailleurs, les manuels indiquent généralement que l’Europe a été fondée pour rivaliser sur le plan économique et industriel avec la puissance des États-Unis et de l'URSS ${ }^{52}$, et plusieurs manuels soulignent le fait que la France sous la Ve République maintient son indépendance en s'opposant parfois aux décisions prises par les Etats-Unis ${ }^{53}$. Surtout, les manuels expriment de la fierté pour la richesse et le prestige de la France dans les domaines culturel, intellectuel et humanitaire. Par exemple, Hatier affirme :

«Les dirigeants de la France ont agi pour que le pays conserve sa place dans les relations internationales et pour qu'il soit reconnu comme la "patrie des droits de l'homme"54. »

Par ailleurs, ce manuel explique :

«La culture américaine influence l'ensemble de la planète: partout, on porte des jeans, on écoute des musiques américaines et on regarde des films et des séries venus des États-Unis, on mange des hamburgers. L'anglais sert de langue de communication internationale. Les autres influences culturelles sont plus spécifiques: mangas et sushis japonais, pizzas italiennes, cuisine chinoise, musique brésilienne... La France est connue pour son patrimoine, ses idées (la «patrie des droits de l'homme»), sa gastronomie, sa mode ${ }^{55}$...»

Ce texte réducteur envers les contributions culturelles de plusieurs pays, présentées comme étant uniquement d'ordre matériel, souligne par contraste la nature noble et supérieure de la France. Il s'agit là d'un procédé classique pour définir un groupe ou une communauté: se distinguer des autres et affirmer sa propre supériorité. Face à la crise perçue (par une grande partie du public) de l'identité nationale ${ }^{56}$, certains manuels français reprennent, en effet, des méthodes qui insistent sur la supériorité morale et culturelle du peuple français. Le nationalisme est ainsi associé à la culture matérielle, intellectuelle, artistique et politique de la France, y compris une affirmation de l'importance de la solidarité face au capitalisme mondial.

En revanche, les manuels américains, conformément aux programmes d'États comme le Texas, montrent le capitalisme sous un jour favorable, en le présentant comme une des grandes libertés dont bénéficient les Américains. Cette tendance est nouvelle. Les programmes du Texas exigeaient déjà au début des années 1980 que soient enseignés « les bienfaits du système du libre marché ». Dans la pratique, les ouvrages des années 1980 et 1990 contenaient se limitaient à quelques allusions éparses sur le développement du système « du libre marché » à l'époque coloniale et sur l'intérêt que ce système présente pour les immigrés. Les ouvrages de 2003 abordent très différemment ce sujet, en y consacrant entre deux et quatre pages, avec des images valorisant la société d'abondance, et un texte en soulignant ses avantages pour les consommateurs, pour l'innovation scientifique et technique, pour faire fortune, et surtout pour choisir librement sa voie. Par exemple, dans sa présentation

\footnotetext{
51 Par exemple, Hatier CM2 2010, p. 179 ; Sedrap CM2 2010, p. 125. Ce constat rejoint les conclusions de Vincent Martigny concernant l'anti-américanisme et le nationalisme culturel français: Vincent Martigny, Le nationalisme culturel..., op. cit., p. 447-540.

52 Par exemple, Sedrap CM2 2010, p. 68.

53 Hatier CM2 2010, p. 89.

54 Ibid.

55 Ibid., p. 175.

56 Gérard Noiriel, À quoi sert..., op. cit. ; Elaine Thomas, Immigration, op. cit.
} 
Rachel Hutchins, "Construction et négociation de l'identité nationale. Une étude comparatiste des programmes et des manuels scolaires d'histoire pour le primaire en France et aux États-Unis », Histoire@Politique, n ${ }^{\circ} 26$, maiaoût 2015, www.histoire-politique.fr

du sujet, un ouvrage utilise les mots «liberté » ou «libre » trente fois en quatre pages $^{57}$. Ces ouvrages évoquent le mythe de l'égalité des chances ${ }^{58}$, parfois de manière très explicite: « Ce système donne l'opportunité égale d'être propriétaire et de gérer sa propre entreprise ${ }^{59}$. »

Dans cette optique, ces ouvrages montrent également la mondialisation sous un jour favorable, comme un libre échange au bénéfice de tous. Par exemple, un ouvrage de 2003 explique :

« Le commerce international ajoute beaucoup à l'économie des États-Unis. Les ÉtatsUnis agissent également par d'autres moyens avec les autres pays. De nombreuses entreprises américaines ont des bureaux et des usines dans d'autres pays. Beaucoup d'entreprises des autres pays ont aussi des sociétés aux États-Unis. Presque cinq millions de personnes aux États-Unis travaillent dans des sociétés appartenant à des personnes d'autres pays. Ceci signifie que les nations du monde font désormais partie d'une économie mondiale $[\ldots]^{60}$. »

Alors que les manuels français se terminent généralement sur le sujet des inégalités et de la solidarité nécessaire pour y remédier, les ouvrages américains les plus récents concluent avec des assertions sur le patriotisme. Ce choix contraste avec les manuels des années 1980 et 1990 qui s'achevaient sur les défis à relever pour les États-Unis ; les élèves étaient alors investis de la responsabilité de relever ces défis pour le bien du pays et du monde. Si certains aspects de ce récit persistent dans les manuels les plus récents, ils n'en sont plus la note finale. Ces manuels s'achèvent tous sur l'importance du patriotisme pour assurer l'avenir du pays :

«Des républiques du passé, comme celle de la Rome antique, se sont dissoutes en partie parce que le peuple est devenu avare et égoïste. Pour que la nation reste forte, les Américains devront conserver l'esprit qui a donné à la nation son indépendance et sa Constitution. Ils doivent faire preuve de patriotisme, ou l'amour de son pays. Patriotisme signifie plus que simplement brandir le drapeau américain à des moments exceptionnels. Les rédacteurs de la Constitution savaient que les Américains devraient être bons citoyens tout le temps ${ }^{61}$. »

Par leur présentation des sujets délicats concernant les femmes, les minorités ethniques, et les inégalités socio-économiques, les manuels américains font preuve d'un retour vers des éléments d'une version archaïque de l'histoire nationale. Cette vision du passé national souligne l'unité en limitant la visibilité des divisions et des conflits sociaux. Si les femmes et les minorités ethniques sont assez visibles dans ces ouvrages, il s'agit avant tout d'une sorte de multiculturalisme de compromis, où l'on se félicite de la diversité de la population et de la culture à travers un discours de

\footnotetext{
57 Scott Foresman 2003, p. 18-21.

58 L'idée (démentie par les statistiques concernant la pauvreté [Garth L. Mangum, Stephen L. Mangum et Andrew M. Sum, The Persistence of Poverty in the United States, Baltimore, Johns Hopkins University Press, 2003]) selon laquelle la réussite économique serait à la portée de tous, à condition de travail acharné et grâce au système capitaliste américain, est l'un des mythes fondateurs des États-Unis (Elaine Marienstras, Les Mythes fondateurs..., op. cit., p. 295) et fait encore partie des convictions d'une majorité d'Américains (voir par exemple Janny Scott et David Leonhardt, " Shadowy Lines That Still Divide », The New York Times, 15 mai 2005, http:/ / www.nytimes.com/ 2005/ 05/ 15/ us/ class/ shadowylines-that-still-divide.html (consulté le 11juin 2005).). Alors que les ouvrages des années 1980 démentaient ce mythe, ceux de 2003 le véhiculent.

59 McGraw-Hill 2003, p. 26.

60 Harcourt 2003, p. 636.

61 Harcourt 2003, p. 643.
} 
Rachel Hutchins, "Construction et négociation de l'identité nationale. Une étude comparatiste des programmes et des manuels scolaires d'histoire pour le primaire en France et aux États-Unis », Histoire@Politique, n ${ }^{\circ} 26$, maiaoût 2015, www.histoire-politique.fr

progrès commun continuel vers l'égalité, tout en minorant les questions plus polémiques de la persistance de la discrimination et de ses conséquences. Le racisme apparent qui caractérisait encore les manuels d’histoire dans les années 1950 a été éradiqué des ouvrages contemporains, et cela représente un véritable progrès. Néanmoins, en évitant d'étudier les conséquences du racisme dans l'histoire américaine, les manuels scolaires trouvent peut-être un moyen de satisfaire la classe blanche centriste ou conservatrice; mais cela se fait au détriment d'une grande partie de la population américaine.

Cette évolution, qui s'est amplifiée après les années 1990 et l'accroissement du pouvoir politique (au sens large) des conservateurs texans dans le domaine de l'enseignement ${ }^{62}$, va de pair avec un éloignement des idéaux de justice sociale. Ainsi, les manuels américains les plus récents - contrairement à leurs prédécesseurs des années 1980 et 1990 - mettent l'accent sur un patriotisme fondé sur l'appréciation du capitalisme et du statu quo. Ces ouvrages, encore plus que les programmes sur lesquels ils se fondent, visent davantage à transmettre la mémoire nationale qu'à enseigner l'histoire en tant que discipline scolaire.

Les manuels et les programmes français abordent davantage l'histoire nationale en rapport avec l'histoire savante. Cette différence reflète sans doute en partie les processus différents de rédaction des programmes et des manuels, l'enseignement français étant davantage façonné par des historiens que par des notions populaires du passé national.

Les différences institutionnelles n'expliquent toutefois pas toutes ces différences idéologiques. Au-delà de la dimension forcément nationaliste (dans le sens retenu ici) de l'enseignement de l'histoire dans un cadre essentiellement national, les programmes et les manuels français, à l'instar des ouvrages américains, font preuve de nationalisme: ils prônent une vision de la France fière de son rayonnement culturel et de ses valeurs, notamment son engagement pour l'égalité sociale et la solidarité. En adoptant une approche plus critique de l'histoire et de la société françaises, les programmes et les manuels français se montrent fiers de cette capacité d'autocritique et de la tradition intellectuelle dont elle découle, autocritique qui est l'un des fondements d'une politique de justice sociale et de l'identité nationale française telle que ces ouvrages la représentent. Cette image de la France reflète ainsi un nationalisme de gauche ${ }^{63}$, dont les fondements sont issus de la Révolution française, et dont les visées républicaines d'égalité (définie en des termes socioéconomiques), s'alignent avec une pédagogie historique critique. Bien qu'il existe des Américains qui se reconnaissent dans cette conception du rôle de l'État, l'enseignement de l'histoire aux élèves du primaire continue de promouvoir le mythe de la supériorité américaine. Une telle conception de l'identité nationale ne peut qu'être associée à une pédagogie mémorielle et triomphaliste ${ }^{64}$.

\footnotetext{
62 Rachel Hutchins, «Heroes and the Renegotiation of National Identity in American History Textbooks : Representations of George Washington and Abraham Lincoln, 1982-2003 », Nations and Nationalism, tome 17, $\mathrm{n}^{\circ}$ 3, 2001, p. 649-668.

63 J.J. Schwartzmantel, «Class and Nation: Problems of Socialist Nationalism », Political Studies, tome XXXV, 1987, p. 239-255.

64 D'autres études montrent que le même état des faits caractérise l'enseignement secondaire aux ÉtatsUnis. Voir par exemple: James Loewen, Lies..., op. cit. ; B. VanSledright, « Narratives...», op. cit., p. 109-146. J onathan Zimmerman, Whose America ? Culture Wars in the Public Schools, Cambridge MA ; London, Harvard University Press, 2002.
} 
Rachel Hutchins, «Construction et négociation de l'identité nationale. Une étude comparatiste des programmes et des manuels scolaires d'histoire pour le primaire en France et aux États-Unis », Histoire@Politique, n ${ }^{\circ} 26$, maiaoût 2015, www.histoire-politique.fr

En dépit de ces différences du caractère de l'identité nationale, en France comme aux États-Unis, l'enseignement de l'histoire et les polémiques autour de l'identité nationale qui ont influencé les programmes depuis les années 1980 participent à une réaffirmation et à une redéfinition de la nation et de ses valeurs. Bien que les résultats diffèrent sur le plan idéologique, ce processus est avant tout nationaliste, car c'est au travers des débats sur qui «nous » sommes, ce en quoi « nous » croyons que la communauté nationale est renforcée, régénérée, et définie comme étant une communauté65. Dans le domaine de l'éducation, l'enjeu est double: la communauté de citoyens qui débat de l'identité nationale resserre ainsi ses liens et, en forgeant une nouvelle définition de la nation, (ré)affirme l'attachement des participants de ces débats à la nation. Par ailleurs, la vision du passé et de la société actuelle que tentent de transmettre les programmes et les manuels scolaires vise à ancrer la nation dans le passé et dans le futur de la prochaine génération de citoyens.

Alors que de nombreux chercheurs dans plusieurs disciplines réclament l'enseignement d'une histoire scolaire cosmopolite afin de transmettre des sentiments d'appartenance au-delà de l'État-nation ${ }^{66}$, l'engagement nationaliste de l'histoire scolaire en France et aux États-Unis ne fait que se réaffirmer. Ce constat confirme l'idée selon laquelle tout ce qui semble menacer la nation, que ce soit la perte de pouvoir politique en faveur des instances supranationales ou la possibilité de changement du caractère national lié aux évolutions démographiques, a tendance à renforcer la cohésion nationale et l'intensité des expressions du nationalisme ${ }^{67}$. Ainsi, à travers l'enseignement scolaire de l'histoire, la nation se perpétue en tant que communauté politique et affective de référence.

\section{Corpus étudié}

\section{Manuels scolaires français}

- Gilles Baillat et al., 1996. Histoire, géographie, éducation civique: Cycle 3, niveau 3, coll. « Gulliver », Paris, Nathan.

- André Bendjebbar et al., Multi-livre CM1: Histoire, géographie, sciences, Paris, Istra, 2003.

- Patrick Beyria et al., À nous le monde! Cycle 3, 2e année, CM1: Histoire, géographie, sciences, éducation civique. Paris, Sedrap, 1998.

- Patrick Beyria et al., À nous le monde! Cycle 3, 2e année, CM1: Histoire, géographie, sciences, éducation civique, Paris, Sedrap, 2003.

- Patrick Beyria et al., À nous le monde! Cycle 3, 3e année, CM2 : Histoire, géographie, sciences, éducation civique, Paris, Sedrap, 2003.

- Sandra Boëche (dir.), CM1 : Les Reporteurs histoire, géographie, histoire des arts, développement durable, Paris, Sedrap, 2011.

\footnotetext{
65 J ohn Hutchinson, Nations as Zones of Conflict, op. cit.

66 Voir par exemple, Kevin McDonough et Walter Feinberg (eds.), Citizenship and Education in LiberalDemocratic Societies, Oxford, Oxford University Press, 2003. Martha Nussbaum, « Patriotism and Cosmopolitanism », Boston Review, oct./nov. 1994, http://bostonreview.net/martha-nussbaumpatriotism-and-cosmopolitanism (consulté le 1er mars 2015) ; Hanna Schissler, Yasemin Nuhoglu Soysal (eds.), The Nation, Europe, and the World: Textbooks and Curricula in Transition, New York; Oxford, Berghahn Books, 2005.

67 Gil Delannoi, « La Théorie de la nation et ses ambivalences », dans Gil Delannoi et Pierre-André Taguieff, Théories du nationalisme, Paris, Kimé, 1991, p. 14 ; Eric Hobsbawm, Nations and Nationalism since 1780: Programme, Myth, Reality, Cambridge, Cambridge UP, 1992, p. 120-121; 170-171.
} 
Rachel Hutchins, "Construction et négociation de l'identité nationale. Une étude comparatiste des programmes et des manuels scolaires d'histoire pour le primaire en France et aux États-Unis », Histoire@Politique, n 26, maiaoût 2015, www.histoire-politique.fr

- Sandra Boëche (dir.), CM2 : Les Reporteurs histoire, géographie, histoire des arts, développement durable, Paris, Sedrap, 2011.

- J ean-Pierre Chevalier et al. (dir.), Histoire, histoire des arts, instruction civique, CM2, Paris, Nathan, 2010.

- Gracia Dorel-Ferré et M. Dhainaut, Cours moyen : Histoire, Paris, Armand Colin, 1985.

- Gracia Dorel-Ferré et al., Histoire, géographie, CM2 Cycle 3: Une Terre, des hommes, Paris, Magnard écoles, 1998.

- Roselyne Le Bourgeois, Histoire, histoire des arts, CM1, Paris, Nathan, 2010.

- Sophie Le Callennec et al., Histoire-géographie, Cycle3, niveau 2, CM1, Paris, Hatier, 1997.

- Sophie Le Callennec et al., Histoire-géographie, Cycle3, niveau 3, CM2, Paris, Hatier, 1998.

- Sophie Le Callennec et al., Histoire, géographie, CM2, Cycle 3, Paris, Hatier, coll. « Magellan », 2004.

- Sophie Le Callennec et al., Éducation à la citoyenneté, Cycle 3, Paris, Hatier, coll. « Magellan », 2008.

- Sophie Le Callennec et al., Histoire, géographie, CM1, Paris, Hatier, coll. « Magellan », 2010.

- Sophie Le Callennec et al., Histoire, géographie, CM2, Paris, Hatier, coll. « Magellan », 2010.

- Hugues Lécharny et al., Histoire géographie CM1 Cycle3, Paris, Nathan, coll. « Vers le monde », 2007.

- J ean-Louis Nembrini, Pierre Polivka, et J ean Bordes, Histoire CM, Paris, Hachette écoles, coll. « Pour connaître la France», 1985.

- J ean-Louis Nembrini, Pierre Polivka, et J ean Bordes, Géographie CM, Paris, Hachette écoles, coll. « Pour connaître la France», 1985.

- Marc Vincent et al., CM1: Histoire: La France au fil du temps, de la Préhistoireà 1789, Paris, Nathan, coll. « Télémaque », 1985.

- Marc Vincent et al., CM2 : Histoire: La France au fil du temps, de 1789 à nos jours, Paris, Nathan, coll. « Télémaque », 1985.

\section{Manuels scolaires américains}

- Val E. Arnsdorf et al. The United States and Its Neighbors. Morristown, NJ : Silver Burdett, 1982.

- James. A. Banks et al. United States: Adventures in Time and Place. New York: Macmillan McGraw-Hill, 1997.

- J ames. A. Banks et al. Our Nation. New York: Macmillan McGraw-Hill, 2003.

- Sarah Bednarz et al. Build Our Nation. We the People. Boston, MA: Houghton Mifflin, 1997

- Michael Berson et al. Harcourt Horizons: United States History. Orlando, FL: Harcourt, 2003.

- Richard G. Boehm et al. America's Story: Stories in Time. New York: Harcourt-Brace, 1997.

- Candy Dawson Boyd et al. The United States, Social Studies. Glenview, IL: Scott Foresman, 2003.

- Juan. R. Garcia et al. Our United States: Social Studies. Parsippany, NJ : Silver Burdett Ginn, 1997.

- Herbert Gross et al. Exploring Our World: Americas (2nd edn). Chicago, IL: Follett, 1980.

- Allen Y. King et al. The United States and the Other Americas. New York: Macmillan. 1982. 
Rachel Hutchins, "Construction et négociation de l'identité nationale. Une étude comparatiste des programmes et des manuels scolaires d'histoire pour le primaire en France et aux États-Unis », Histoire@Politique, n² 26, maiaoût 2015, www.histoire-politique.fr

- Frederick M. King et al. Understanding Our Country. River Forest, IL: Laidlaw, 1981.

- Stanley Klein et al. Our Country's History: Level E. New York: Scholastic, 1982

\section{Programmes et documents officiels}

- “Chapter 113: Texas Essential Knowledge and Skills for Social Studies.” 1998. Texas Essential Knowledge and Skills. Texas Education Agency. Available at : http:// www.tea.state.tx.us/rules/tac/ ch113.html\#s113 (consulté le 20 déc. 2000).

- "Chapter 113: Texas Essential Knowledge and Skills for Social Studies." 2010. Texas Essential Knowledge and Skills. Texas Education Agency. Available at : http:// ritter.tea.state.tx.us/rules/tac/ chapter113/index.html (consulté le 5 nov. 2013).

- Framework for the Social Studies. 1980. Austin: Texas Education Agency.

- "Horaires et programmes d'enseignement de l'école primaire", Bulletin officiel de l'Éducation nationale, 2002, numéro spécial 1 (14 fév.).

- "Horaires et programmes d'enseignement de l'école primaire", Bulletin officiel del'Éducation nationale, 2008, numéro spécial 3 (19 juin).

- "Horaires, programmes et instructions pour l'école élémentaire, 1985", Bulletin officiel de l'Éducation nationale, 1986, numéro spécial 1 (16 jan.).

- Ministère de la Jeunesse, de l'Éducation nationale et de la Recherche: Direction de l'enseignement scolaire, "Histoire et géographie cycle des approfondissements (cycle 3)", Paris, Centre national de documentation pédagogique, coll. «École: Documents d'application des programmes », 2002.

- Proclamation 1994 of the State Board of Education Advertising for Bids on Instructional Materials. 1994. Austin: Texas Education Agency.

- "Programmes de l'école primaire", Bulletin officiel de l'Éducation nationale, 1995, n 48 (28 déc.).

\section{L'auteur}

Rachel Hutchins est maître de conférences en civilisation américaine à l'Université de Lorraine. Sa monographie Nationalism and History Education: Curricula and Textbooks in the United States and France sortira chez Routledge début 2016.

\section{Résumé}

À la lumière des débats concernant l'identité nationale en France et aux États-Unis, cet article cherche à élucider les objectifs nationalistes (au sens développé dans l'article) de l'enseignement de l'histoire dans les deux pays. Il démontre comment les réécritures de l'histoire scolaire reflètent et contribuent à redéfinir la nation et, par ce biais, la perpétuent. Il examine la forme que prend le nationalisme dans les deux pays et les valeurs qu'il véhicule, révélant, entre autres, une approche française qui applique des méthodes inspirées des travaux d'historiens et vise à promouvoir la solidarité, tandis que les manuels américains, sous l'impulsion des mouvements conservateurs des trente dernières années, prônent une vision de la mémoire nationale fondée sur une unité fantasmée.

Mots clés : identité nationale, nationalisme, enseignement, histoire, France, ÉtatsUnis

\section{Abstract}


Rachel Hutchins, "Construction et négociation de l'identité nationale. Une étude comparatiste des programmes et des manuels scolaires d’histoire pour le primaire en France et aux États-Unis », Histoire@Politique, n 26, maiaoût 2015, www.histoire-politique.fr

In the light of debates over national identity in France and the United States, this article seeks to elucidate the nationalist objectives of history education in both countries. The article demonstrates how rewriting history for schools reflects and contributes to redefining the nation and, in this manner, perpetuates the nation. The article examines the shape that nationalism takes in both countries and the values that it promotes, revealing, among other conclusions, that the French textbooks adopt more historians' methods and aim more to promote solidarity, whereas the American textbooks - under the influence of conservative movements of the past thirty years advance a vision of national memory founded on a myth of unity.

Keywords : National Identity; Nationalism; Education; History; France, United States.

Pour citer cet article : Rachel Hutchins, « Construction et négociation de l'identité nationale. Une étude comparatiste des programmes et des manuels scolaires d'histoire pour le primaire en France et aux États-Unis », Histoire@Politique, n² 26, mai-août 2015, www.histoire-politique.fr 